\title{
Gobierno y sociedad en un núcleo urbano de la Rioja Baja: Calahorra (s. XIII-inicios del XVI)
}

\author{
FCO. JaVier Goicolea JuLian \\ Universidad de La Rioja
}

\begin{abstract}
RESUMEN
En este trabajo se estudia la organización concejil y social de

Calahorra, con objeto de poder observar en la medida que la documentación utilizada lo ha permitido, las transformaciones que tuvieron lugar en la organización del poder municipal y de la sociedad política de esta urbe riojana desde la plena Edad Media hasta principios del siglo XVI. No cabe duda de que esas transformaciones, junto a las no menos importantes pervivencias en la organización socio-política de la ciudad, forjaron

el gobierno y la sociedad urbanas de la Calahorra de los primeros

ABSTRACT

In this work, the council and social organization in Calahorra is studied to observe, as long as in has been possible by the documentation available, the changes that took place in the organization of the municipal power and in the political society of this Riojan city, from the central period of the Middle Ages to the beginning of the XVI century. There is no doubt that these changes, together with the not less important survivals in the sociopolitical organization of the city, built up the urban government and society of Calahorra during the first years of the Modern Age.
\end{abstract} años de la Edad Moderna.

PALABRAS CLAVE

Rioja Baja, Calahorra, sociedad, poder municipal, siglo XIII - inicios siglo XVI.

KEYWORDS

Low Rioja, Calahorra, society, local power, XIII century - beginning XVI century

\section{INTRODUCCIÓN}

A fines del siglo XI Nájera y Calahorra constituian las principales referencias urbanas del espacio territorial riojano, aunque los papeles ejercidos por ambos centros de poder en este territorio fueran distintos. De esta forma, si en el caso de Nájera su función articuladora del espacio altorriojano se pone claramente de manifiesto en la documentación de esta época, en Calahorra 
el papel articulador con respecto a La Rioja Baja es menos explícito en los documentos. Efectivamente, tal y como afirma el profesor José Ángel García de Cortázar, el florecimiento de Calahorra tendrá que ver menos con los progresos de una organización del espacio protagonizada internamente por la sociedad asentada en La Rioja, que con su papel de plaza fronteriza dentro del reino de Castilla'.

Los monarcas castellanos, muy interesados en dominar el territorio riojano, reconocieron mediante privilegios el importante protagonismo de Calahorra en la defensa de la frontera ${ }^{2}$, lo que unido a su condición de sede obispal, favoreció el progresivo crecimiento de la urbe a lo largo de los siglos medievales y la consolidación en Calahorra de una sociedad estructurada en líneas generales en estados o estamentos, y en la que el clero tenía asimismo un destacable protagonismo ${ }^{3}$. A fines de la Edad Media e inicios de la Moderna, por lo tanto, Calahorra era una de los núcleos urbanos más importantes de La Rioja, aunque desgraciadamente el nivel de importancia de la ciudad calagurritana no esté acorde con el nivel de conocimiento que poseemos sobre su organización interna en el período de tiempo propuesto. Por ello, con la presente investigación pretendemos realizar una primera aproximación a la organización concejil y social de Calahorra desde el sigio XIII, aunque prestando especial atención a los años finales del siglo XV e inicios del XVI, y para este objetivo nos valdremos tanto de fuentes documentales ya publicadas como de documentos inéditos procedentes de diversos archivos ${ }^{4}$.

\section{LOS ANTECEDENTES: EL GOBIERNO CONCEJIL EN LOS SIGLOS XIII Y XIV}

La documentación calagurritana del siglo XIII nos muestra la presencia mayoritaria de tres alcaldes al frente del concejo urbano, si bien, este número varió en el período comprendido entre los años 1232-1246, cuando ejercieron sus funciones cuatro alcaldes concejiles ${ }^{5}$. Junto a estos alcaldes,

"José Ángel GaRcia de CORTAZAR, "La organización social del espacio riojano en vísperas de la con. cesión del Fuero de Logroño", Actas de la Reunión Científica El Fuero de Logroño y su época, Logroño, 1996, pp. 191-207.

En 1255 Alfonso X concedió licencia al concejo de Calahorra para celebrar cmercado cada semana en el dia de miercoles" (Enrique CANTEFA MONTENEGRO, "Franquicias regias a ciudades y villas riojanas en el marco de la política repobladora de Altonso X", Berceo, n. ${ }^{\circ} 114-115$ (1988), pp. 105-118, y A.M.C... Privilegios, relación de privilegios concedidos a Calahorra desde el año 1255).

Según las ordenanzas confeccionadas en 1515 para regular el trabajo de los cinco alcaldes del campo de Calahorra, uno de estos alcaldes debia ser elegido por el cabildo catedral de la ciudad.

${ }^{4}$ Concretamente de los archivos de la Real Chancillería de Valladolid (A.R.CH.V.), General de Simancas (A.G.S.) y Municipal de Calahorra (A.M.C.).

${ }_{5}$ Ildefonso Rodriguez de Lama, Colección Diplomática Medieval de la Rioja. Tomo III-documentos (1168-1225), Logroño, 1979 y Colección Diplomática Medieval de la Rioja. Tomo IV-documentos siglo XIII, Logroño, 1989. 
también documentamos la presencia de un juez y un sayón, además de los jurados ( 3 en 1243 y 4 en 1267), oficiales que cobraron especial importancia a partir de la aplicación del Fuero real, puesto que fueron ellos los que conformaron el organismo de hombres buenos encargado de auxiliar a los alcaldes, tal y como se especificaba en la reforma alfonsina ${ }^{6}$.

El gobierno concejil de Calahorra se iba consolidando, por lo tanto, a lo largo del siglo XIII, aunque la influencia del poder real en la vida política municipal se seguía poniendo igualmente de manifiesto en el núcleo urbano, sobre todo en determinados años en los que se documenta la presencia de alcaldes del rey y jurados del rey en Calahorra ${ }^{7}$. No hay que olvidar, además, que hacia mediados del siglo XIII el monarca Alfonso X trató de intervenir en los gobiernos municipales con objeto de reformarlos, aplicando para ello su proyecto ya citado de unificación de los derechos locales mediante el Fuero real. En La Rioja, se sabe que los concejos de Logroño y Santo Domingo de la Calzada mantuvieron una actitud negativa ante el Ordenamiento, ejerciendo una fuerte resistencia a su implantación, de manera que su aplicación no fue rigurosa, concretándose en el establecimiento de un esquema básico de gobierno urbano que se adecuó a cada una de las coyunturas locales ${ }^{8}$.

Este esquema de gobierno concejil quedó concretado en Calahorra mediante la confirmación en sus principales puestos a alcaldes y jurados, quienes se reunían en sesiones concejiles que eran pregonadas por el oficial pregonero la noche anterior y por la mañana. Estas sesiones concejiles solían tener lugar en la capilla de San Juan de la iglesia catedral de Santa María, interviniendo en ellas los principales magistrados municipales, pero también otros vecinos, puesto que se mantenían las sesiones de concejo abierto $^{9}$. En este sentido, la representación política vecinäl de Calahorra se estructuraba a partir de tres collaciones, que elegian a sus representantes con objeto de defender sus intereses, y realizaban asimismo sus propias asambleas de collación pregonadas por la noche y por la mañana. Un documento de principios del siglo XIV, concretamente de 1318, permite constatar que este tipo de articulación política vecinal se mantenía en la centuria del trescientos. Ciertamente, en 1318 se documentan dos representantes, procuradores o voceros de la collación de Media Villa en la asamblea de esta

\footnotetext{
"Pascual MARTinez SOPENA, "Logroño y las villas riojanas entre los siglos XII y XIV", Historia de la ciudad de Logroño, vol. Il (Edad Media, José Ángel Sesma Muño: , Coord.), Logroño, 1995, pp. 279-322.

Ildefonso Rodriguez de Lama, Colección Diplomática Medieval de la Rioja. Tomo IV-documentos siglo XIII..., o.c., doc. n. ${ }^{\circ} 119$ (año 1237).

- Pascual Martinez SOPENA, "Logroño y las villas riojanas entre los siglos XII y XIV..., o.c., pp. 302-304.

- El 11 de abril de 1237, sábado de Ramos, fue realizada una concordia entre al concejo de Calahorra y los "frayres" de la aldea de Casanueva, con objeto de reglamentar el aprovechamiento de unos pastos. Pues bien, en el documento se señala que se reunió “todo conceio de Calaforra asi todos los mayores como los menores" (Ildefonso Rodriguez DE LAmA, Colección Diplornática Medieval de la Rioja. Tomo IV-documentos siglo XIII..., o.c., doc. n. $\left.{ }^{0} 119\right)$.
} 
collación, junto a un alcalde concejil ${ }^{10}$; mientras en 1361 podemos constatar la presencia de varios procuradores de las collaciones de Media Villa y de Barrio Suso, que se reunieron con los representantes del cabildo catedral de Calahorra con objeto de acordar en qué lugar se debían celebrar los mercados de los miércoles ${ }^{11}$.

Si nos atenemos a las relaciones de oficiales concejiles de Calahorra a lo largo del siglo XIII, se puede comprobar asimismo que determinados individuos ejercieron alcaldías y juradurías durante un número considerable de años consecutivos. En efecto, entre otros miembros de la sociedad política local de Calahorra en la centuria del doscientos, se documentan personajes como Pedro Zahet, alcalde entre 1202 y 1212, Miguel de Pedro Zahet, alcalde entre 1219 y 1234, lohannes de Dominico Sendovan, alcalde entre 1224 y 1228, don Mateo, alcalde entre 1257 y 1263, o Garci Sánchez de Entrena, alcalde entre 1284 y $1299^{12}$. Se trataba de miembros de la élite social ciudadana del núcleo urbano, personajes pertenecientes a familias de comerciantes, cambistas, tenderos y prestamistas vinculados a la monarquía, y que desde muy pronto mostraron un gran interés por acumular patrimonio rústico, convertirse en gestores del realengo y alcanzar una condición privilegiada para sus propiedades. Para ello, una de las fórmulas utilizadas por estos personajes fue la concertación de matrimonios con miembros de familias de la aristocracia local riojana, que como en la caso de algunos miembros de la familia Zapata (alcaides de Calahorra en la segunda mitad del siglo XII), estaban interesados a su vez en participar en el gobierno municipal de Calahorra con objeto de mejorar su posición social e incrementar su patrimonio. Efectivamente, sabemos que Martín Zapata contrajo matrimonio con Urraca Sánchez, hija de un miembro del concejo calagurritano, y asimismo tenemos constancia del nombramiento como alcaldes de miembros de la familia Zapata; entre ellos, Pedro Zapata (1258-1260) y un nieto de Jimeno Zapata llamado Esteban de Doña Eva (1240-1262) ${ }^{13}$.

"In Dei Nomine. Nos Rroy Sanchez e Pero Garçeyz en boç de la collaçion de Medya Villa sseyendo pregonada de noch e de mañana e sseyendo present el alcalle don Martyn a rrazon de dineros que debe del pecho luan Martinez Felizes vendemos una su pieça en El Pinadera de Soruan a vos Rroy Gonçalez conpanero de la eglesia de Santa Maria por CXXV mrs alffonsis con su yantar " (Eliseo Sáinz Ripa y Venancio Hernaez Iruzubieta, Documentación Calagurritana del siglo XIV. Archivo Catedral, Logroño, 1995, vol. I, doc. n. ${ }^{.76}$ ).

Como procuradores de la collación de Media villa intervinieron Sancho Ferrandez, hijo de Gil Ferrandez, y Sancho Garceiz, hijo de Juan Sancho, mientras en representación de la collación de Barrio Suso, intervineron Juan Diaz de Gauna. Gonzalo Martínez herrero y Pedro Fernández, hijo de Garci Fernández (Eliseo Sainz RIPA y Venancio Hernaez IRUzubieta, Documentación Calagurritana del siglo XIV. Archivo Catedral, Logroño, 1995, vol. Il, doc. n. ${ }^{0} 346$ ).

"Ildefonso Rodriguez de Lama, Colección Diplomática Medieval de la Rioja. Tomo III-documentos (1168-1225), doc. $n^{\circ} 404$ y ss., y Colección Diplomática Medieval de la Rioja. Tomo IV-documentos siglo $X I I I$.., o.c., doc. $n 17$ y ss.).

Tomás SAÉNZ DE HARO, "Los Zapata (1148-1340). Un ejemplo de aristocracia local en La Rioja Baja durante la Edad Media", Los espacios del poder en la España medieval (Actas de la XII Semana de Estudios Medievales, Nájera 2001), Logroño, 2002, pp. 553-582. 
Mientras tanto, a inicios del siglo XIV la política municipal impulsada por Alfonso $X$, fue retomada con nuevos bríos durante el reinado de Alfonso XI, especialmente a partir de la mayoría de edad de este monarca. Entre otras medidas, se favorecía desde el poder real la incorporación de aldeas a los alfoces concejiles y se daba una respuesta positiva a las demandas de mayor autonomía del poder concejil. Así se puede comprobar en 1326, cuando a petición del concejo de Calahorra, Alfonso XI le concedió el privilegio de inmunidad de entrada de adelantados y merinos mayores de Castilla, así como de otros oficiales en su nombre, a causa de las quejas por los daños y agravios que causaban las intervenciones de estos oficiales regios en Calahorra ${ }^{14}$. Varios años después, en 1335, el adelantado mayor de Castilla se comprometía ante el concejo calagurritano a respetar el privilegio otorgado por Alfonso $\mathrm{Xl}^{15}$, monarca que además incorporó este mismo año el lugar de Murillo a la jurisdicción concejil de Calahorra ${ }^{16}$. Se trataba, en definitiva, de favorecer al poder concejil, aunque interviniendo al mismo tiempo en los asuntos municipales a través de fórmulas ya utilizadas en el siglo XIII, de ahí las referencias a alcaldes del rey que documentamos otra vez en la urbe calagurritana a partir de inicios de la década de los años treinta del siglo $X I^{17}$.

Estas medidas político-institucionales no fueron, sin embargo, más que el precedente de la reforma municipal fundamental del reinado de Alfonso $\mathrm{XI}$; reforma que se redactó a mediados del siglo XIV y que tuvo su eje principal en la creación del Regimiento ${ }^{18}$. Las noticias sobre su aplicación en el territorio riojano son bastante limitadas, aunque para el caso de Calahorra contamos con algunos datos desde la segunda mitad de la centuria del trescientos. En efecto, por lo menos desde el año 1358 constatamos la presencia de regidores en el gobierno municipal de Calahorra. En 1358 ejercian esta magistratura Martín Garceiz, Pedro Sánchez, Miguel García y Ferrand Sánchez, oficiales que compartían el organigrama de cargos municipales de Calahorra con tres alcaldes, juez, merino, voz de concejo y unos jurados relegados ahora al papel secundario de oficiales subalternos de justicia ${ }^{19}$.

Ahora bien, además de la aparición del oficio de regidor, a lo largo del siglo XIV también se pueden vislumbrar algunos otros fenómenos reseñables desde el punto de vista de la organización concejil de Calahorra.

A.M.C.: DOCUMENTACIÓN DIVERSA, 20010012.

A.M.C.: DOCUMENTACIÓN DIVERSA, 20010008.

${ }^{16}$ A.M.C.: PRIVILEGIOS, 19005.

Eliseo Sainz Ripa y Venancio Hernaez Iruzubieta, Documentación Calagurritana del siglo XIV. Archivo Catedral..., o.c., vol. I, doc. n. ${ }^{9} 211$.

${ }^{16}$ José María MONSALVO ANTÓN, "La sociedad política de los concejos castellanos de la Meseta durante la época del régimen medieval. La distribución social del poder". Actas del II Congreso de Estudios Medievales Concejos y Ciudades en la Edad Media Hispánica, Madrid, 1990, pp. 359-413.

${ }^{19}$ Con posterioridad, en 1374,1388 y 1390 , se documentan únicamente tres regidores, por lo que su número debió variar a lo largo de la segunda mitad del siglo XIV (Eliseo Sainz RIPA y Venancio HernaEz IRUzuBIETA, Documentación Calagurritana del siglo XIV. Archivo Catedral..., o.c., vol. II, docs. n. ${ }^{9} 340,357$, 385 y 386 ). 
En primer lugar, hay que señalar que la introducción de la figura del regidor no supuso la desaparición de las sesiones concejiles de carácter amplio y abierto, que continuaron celebrándose durante la baja Edad Media. El 11 de febrero de 1358, por ejemplo, se reunió el concejo de Calahorra "por pregon fecho de ante noche e de mañana segund que lo avemos de uso e de costumbre", estando presentes, además de los oficiales ya citados, "toda la otra comunidat e universidat del dicho conçeio de la dicha çibdat ${ }^{20}$. $Y$ una asamblea similar se documenta el 27 de octubre de 1388, cuando los miembros del cabildo catedral "de la una parte e los alcalles e rregidores e iurados e otros ofiçiales de la dicha çibdat...e otra muchedumbre de ommes buenos e fiiosdalgo e çibdadanos e de iudios e moros por parte de todo el conçeio", se reunieron con objeto de reglamentar varias medias en relación con los precios de los alimentos, celebración de mercados en la ciudad, etc. ${ }^{21}$. Se observa, por lo tanto, una adaptación de la reforma a la realidad socio-institucional de la ciudad, a la vez que un fortalecimiento del poder concejil de Calahorra, reforzado por el hecho de que a la prohibición de entrada del merino mayor en la urbe, se sumó la supresión de la figura del senior o prestamero de la ciudad. Ciertamente, a partir del año 1352 desaparecen de los documentos calagurritanos las menciones a este máximo delegado regio en la urbe, cuya autoridad en la ciudad está documentada a lo largo del siglo XIII y primera mitad del XIV ${ }^{22}$.

En segundo lugar, desde el año 1340 se puede observar que la duración de los principales oficios municipales de Calahorra comenzó a ser anual, siguiendo una tendencia general que aparece ya consolidada en el mundo urbano riojano de finales de la Edad Media ${ }^{23}$. Se rompía, por lo tanto, el carácter vitalicio que parecia caracterizar a las principales magistraturas municipales de Calahorra con anterioridad, aunque ello no supuso un obstáculo para que la élite siguiera controlando el poder. Efectivamente, la intervención directa de los principales magistrados municipales en unos procesos electivos anuales basados en la cooptación, permitió que determinados miembros de la élite accedieran con frecuencia al gobierno municipal. Martín Pérez Rucio, por ejemplo, fue alcalde en 1340, 1344 y 1349; Gonzalo Ruiz ejerció alcaldías en 1342, 1344 y 1346; Ferrand Sánchez Falcón fue regidor en 1388 y 1390; Pedro García Ponz fue alcalde en 1385 y 1388; Gonzalo Martínez Caya ejerció alcaldías en 1359 y 1390; y dos representantes de la familia Martínez Lázaro, Sancho y Gonzalo, ejercieron regidurí-

Ibidem, doc. п. $\div 340$.

Ibídem, doc. n. ${ }^{9} 385$.

22 Ibidem, doc. n. 326 y ss

is Eliseo Sainz RIPA y Venancio Hernaez Iruzueieta, Documentación Calagurritana del siglo XIV. Archivo Catedral.., o.c., vol. I, doc. n. ${ }^{0} 246$ y ss., y vol. II. Sobre la organizacion institucional de los concejos urbanos altorriojanos a fines de la Edad Media, remito a mi artículo "Concejos urbanos en La Rioja Alta a fines del Medievo: Aspectos institucionales y políticos", Historia. Instituciones. Documentos, n. 26 (1999), pp. 233-254. 
as y alcaldías en 1374 y $1390^{24}$. En definitiva, la renovación anual de los oficios concejiles que se puso en marcha, no modificó sustancialmente la situación anterior de control oligárquico del poder, aunque sí alteró la distribución del mismo entre las familias más poderosas de la ciudad, pues a partir de mediados del siglo XIV un mayor número de miembros de la élite pudo optar a ser elegido a alguno de los principales cargos municipales. Ciertamente, a través de los listados de magistrados concejiles se puede comprobar esta situación, y también que la sociedad política local de Calahorra del siglo XIV se seguía nutriendo fundamentalmente de representantes del estado ciudadano (comerciantes y hombres de negocio). Ahora bien, la constatación asimismo de personajes de diferente procedencia social, como los escuderos Gonzalvo Simón de Tarín (1340) y Gonzalo Ruiz (1346 y 1349), al frente de alcaldías municipales, pone de manifiesto igualmente que el poder concejil no estaba totalmente monopolizado por los ciudadanos en esta centuria ${ }^{25}$.

\section{EL GOBIERNO URBANO A FINES DE LA EDAD MEDIA E INICIOS DEL SIGLO XVI: PERMANENCIAS $Y$ TRANSFORMACIONES}

La situación del concejo y de la sociedad política local de Calahorra durante la mayor parte del siglo XV, presenta todavía más interrogantes que en el siglo precedente, de manera que es únicamente a partir de los años finales del cuatrocientos, cuando la documentación permite conocer algunos aspectos de la evolución social e institucional de Calahorra en el tránsito de la Edad Media a la Moderna. Por lo que respecta a los regidores, eran cuatro a fines del siglo $X \mathrm{~V}$, representando a los dos estados en que se estructuraba sociopolíticamente la ciudad. Tres regidurias estaban reservadas al estado ciudadano (es decir, a su élite), ostentando cada uno de estos tres regidores ciudadanos la representación de su collación de procedencia, mientras la élite noble sólo tenía reservada una de las cuatro regidurias ${ }^{26}$. Las alcaldías, por su parte, seguían siendo tres, como en la mayor parte del siglo XIII y en el XIV, y de la misma forma que ocurría con las regidurias, se repartían entre los estados ciudadano y noble, correspondiendo dos alcaldías a los ciudadanos y una a los hidalgos ${ }^{27}$. El estado ciudadano, en consecuencia, seguía manteniendo su preponderancia en un gobierno municipal compartido, por lo menos desde fines de la Edad Media, con los representantes del estamento noble de la ciudad.

"Eliseo Sainz RIPA y Venancio Hernáez IrUZubieta, Documentación Calagurritana del siglo XIV. Archivo Catedral.., o.c., vol. I, doc. $n^{0} 246$ y vol. II, docs. n. ${ }^{2} 255,295,297,311,326,342,357,381,385$ y 386 . Ibidem, vol. I, doc. n. 242 y vol. II, docs. $n .9313$ y 326 .

A.G.S.: CÁMARA DE CASTILLA, Pueblos, leg. 5, doc. n. 2 y A.M.C.: L.A. 1504-1522. Ibidem 
La evolución en el seno de la sociedad política de Calahorra que parecia apreciarse a lo largo de la centuria del cuatrocientos, quedaba, en consecuencia, claramente confirmada un siglo después, tal y como también había sucedido en otros núcleos urbanos riojanos como Logroño o Nájera, donde caballeros, escuderos e hidalgos en general habian ido alcanzando progresivas cotas de poder en el municipio. De igual forma, la continuidad con respecto a la etapa precedente se ponia de manifiesto en el mantenimiento de las sesiones de concejo amplio, donde, junto con los miembros del Regimiento, también solian estar presentes los representantes de las cuadrillas de cada una de las collaciones de Calahorra: San Andrés, Santiago y Media villa. Los diputados elegidos por cada una de las tres cuadrillas podían participar, de esta manera, en determinadas tareas del gobierno urbano, tal y como ocurrió en 1471, cuando junto a los alcaldes ordinarios, regidores y representantes del cabildo catedralicio, participaron en la confección de un capitulado para armar a cuarenta caballeros en la ciudad ${ }^{28}$.

El mantenimiento de sesiones concejiles abiertas, el modelo electivo vigente y la corrupción municipal, eran causa, por otro lado, de una gran conflictividad social e institucional en Calahorra. Se trataba de una situación de inestabilidad que se repetía en otras ciudades y villas riojanas como Logroño, Nájera, Santo Domingo de la Calzada o $\mathrm{Haro}^{29}$, y también en otros núcleos urbanos del norte de la Corona de Castilla, lugares donde no se había producido un cierre definitivo de las sesiones de gobierno municipal con anterioridad a la llegada al poder de los Reyes Católicos ${ }^{30}$. Los representantes y procuradores del común urbano no se cansaban de denunciar los daños que ocasionaban las luchas por el poder y las actuaciones fraudulentas de la élite gobernante, ya que todo ello iba en perjuicio de los intereses del sector social urbano más desfavorecido. En Calahorra, los hombres buenos labradores, los pobres, huérfanos y viudas, se quejaban en 1490 de la política fiscal impuesta por los miembros del Regimiento, política que perjudicaba gravemente a los sectores más desfavorecidos de la ciudad ${ }^{31}$. Algunos años después, en 1496 y 1497 , el procurador de la comunidad y hombres buenos de Calahorra, Pedro de Niño, denunciaba las irregularidades cometidas por los representantes del gobierno municipal ${ }^{32}$. Una de estas denuncias se refería a la corrupción

${ }^{27}$ A.G.S.: R.G.S., fol. 674 (29-X-1475), los Reyes Católicos confirman el Capitulado de 1471

FCO. Javier Goicolea Julian, Haro: Una villa riojana del linaje Velasco a fines del Medievo, Logroño, 1999, "Para la paz y sosiego de la ciudad y gobernación de vosotros: Las ordenanzas de Logroño de 1488", Historia, Instituciones, Documentos, n. 27 (2000), pp. 113-128, “La ciudad de Nájera en el tránsito de la Edad Media a la Moderna: El concejo, el señor y la sociedad política ciudadana", Hispania, n.. 205 (2000), pp. 425-452, "Sociedad y relaciones de poder en una ciudad riojana a fines del Medievo: Santo Domingo de la Calzada", Espacio, Tiempo y Forma, $H^{a}$. Medieval, n. ${ }^{0} 12$ (1999), pp. 243-286.

${ }^{30}$ Regina Polo Martín, El régimen municipal de la Corona de Castilla durante el reinado de los Reyes Católicos (Organización, funcionamiento y ámbito de actuación), Madrid, 1999.

${ }^{31}$ A.G.S.: R.G.S., fol. $181(9-1 X-1490)$.

${ }^{32}$ A.G.S.: R.G.S., fols. 130 (-VII-1496), 167 (23-VII-1496), 107 (19-1-1497), 259 (24-I-1497). 
existente en el proceso de elección de nuevos regidores: "que los regidores que avian de ser para un anno nonbravan los regidores que avian de ser para el otro anno, e que estos que asy nonbravan diz que eran sus hermanos e parientes muy propincuos e que asy se davan los regimientos por fabores e que la çibdad era mal regida e governada". Ante la gravedad de la situación, el 19 de enero de 1497, los Reyes Católicos ordenaron a las autoridades judiciales de la ciudad investigar el asunto y estudiar la conveniencia o no de modificar el sistema electoral tradicional ${ }^{33}$.

Se encomendaba la tarea al corregidor, pues sabemos que, por lo menos desde la década de los ochenta, los Reyes Católicos habían decidido colocar a este magistrado real de forma permanente, al frente de los núcleos urbanos riojanos de Logroño, Calahorra y Alfaro, todos ellos importantes baluartes defensivos en la frontera con el reino de Navarra ${ }^{34}$. Las principales atribuciones de los corregidores eran judiciales, teniendo este magistrado jurisdicción civil y criminal, así como capacidad para ejecutar las sentencias, ya que se les autorizaba a apremiar, castigar, proceder contra los culpables y ejecutar la justicia. Pero, además, los corregidores asumían otro tipo de funciones en el ámbito municipal; entre ellas, la presidencia de las reuniones del concejo, la defensa de la integridad territorial del núcleo urbano, mantenimiento del orden público, etc. En definitiva, el establecimiento del corregidor y su teniente en Calahorra, supuso una importante novedad en el esquema de gobierno urbano tradicional de la urbe, pues el corregimiento desplazó de su lugar de privilegio a los alcaldes ordinarios, cuyas funciones judiciales y de gobierno fueron asumidas por el corregidor y su teniente ${ }^{35}$.

Pero, volviendo de nuevo a la problemática electoral, de la información contenida en el documento de 1497, se desprende que los conflictos venían de años atrás, pues en el diploma se afirma que ante la situación de corrupción municipal que generaba el sistema cooptativo tradicional, los Reyes Católicos habían remitido un nuevo ordenamiento electoral a la ciudad que, no obstante, seguía sin cumplirse en 1497. En efecto, su aplicación contaba con la radical oposición de un sector de la sociedad política local de Calahorra, por lo que los Reyes Católicos encomendaron al corregidor que les informara sobre la conveniencia de poner en práctica el nuevo ordenamiento que, como en Vitoria (1476) o Logroño (1488), estaba fundamentado en la insaculación: "que el primero dia del anno en que se nombran los regidores se oviese de tener en el probeymyento de ellos çierta forma, que el primero dia del anno en cada un anno a hora de la mysa mayor se juntasen el corregidor e regidores e diputados de la dicha çibdad e

A.G.S.: R.G.S., fol. 107 (19-1-1497).

A.G.S.: R.G.S., fol. 305 (9-1-1488)

Ahora bien, los alcaldes ordinarios de Calahorra seguian ejerciendo sus funciones en los momentos de interinidad, normalmente en el periodo de tiempo que transcurría entre la finalización de un corregimiento y la llegada de un nuevo corregidor a la ciudad (A.M.C.: L.A. 1507, sesión del 19 de septiembre) 
echasen suertes qual de ellos eligiria quatro electores, e al que cupiese la suerte los eligiese e que estos dichos quatro electores so çierto juramento nombrasen diezeseys personas cada uno quatro que toviesen cavallos, secretamente non comunycandolo los unos con los otros e se oviesen de echar todos en un cantaro e de alli sacasen los quatro primeros segund que mas largamente se contenya en la dicha carta $" 36$.

El principal escollo era, sin duda, la cuestión del mantenimiento de caballo con su correspondiente armamento, un requisito que cumplían pocos vecinos de la ciudad, hidalgos sobre todo, frente a un número menor de representantes del estado ciudadano. De esta forma, en el mismo documento de enero de 1497, se afirmaba que no llegaban a dieciséis los vecinos de Calahorra que poseían caballos, la mayor parte de los cuales eran hidalgos. Las consecuencias, por tanto, eran evidentes si se aplicaba el nuevo ordenamiento, ya que, si por un lado los que más opciones tendrian para acceder a las regidurías serían los miembros del estado noble; por otro lado, también podrían ser elegidos los cuatro regidores de una misma collación, con lo que se romperia el carácter representativo tradicional del oficio de regidor. Pero, además, los ciudadanos alegaban que una gran parte de los vecinos que poseían caballo "vivian con caballeros", por lo que no iban a defender tanto los intereses de la ciudad y de sus vecinos, como los propios intereses de los caballeros de los que dependian. En definitiva, afirmaban los ciudadanos que la capacitación para acceder al gobierno de Calahorra no se podía medir en ningún caso por la posesión o no de un caballo de guerra. y apelaban asimismo a los daños que sufriría la hacienda regia si se rompía el reparto político estamental entre ciudadanos e hidalgos. En este sentido, señalaban los ciudadanos que la nueva situación quebraría necesariamente la política fiscal tradicional de la ciudad, pues: "los dichos tres regidores de los çibdadanos cada uno en su colaçion diz que tyenen cargo de dar cojedores y fazer coger los reales pechos e los conçegiles, y el de los fijosdalgo de los fijosdalgo e todos juntos de las cosas tocantes a la unyversidad de la dicha çibdad, e sy se oviese de tener la forma de la dicha provysyon podia acaesçer que todos fuesen fijosdalgo o todos çibdadanos e aun de una colaçion e diz que si fuesen todos fijosdalgo non serian tan bien cogidos los pechos e derechos que a nos nos pertenesçen ny serian obligados a entender en ellos porque es contra su costumbre. E diz que sy fuesen çibdadanos no les serian guardadas las libertades e franquezas commo devian a los fijosdalgo, de donde nasçerian muchos pleytos e otros ynconvenyentes e a nos vernia deserviçio e a la dicha çibdad mucho danno,"

Lo cierto es que en febrero de 1498 se seguía sin aplicar el nuevo ordenamiento electoral, por lo que el día 14 de ese mismo mes, sabemos que el escribano Juan Sánchez de Tejada, miembro del estado noble, había insta-

\footnotetext{
A.G.S.: R.G.S., fol. 107 (19-I. 1497).

si lbídem.
} 
do al teniente de corregidor a poner en marcha la reforma, a lo que este último se negaba por hallarse fuera de la ciudad el corregidor ${ }^{38}$. La cuestión electoral, por lo tanto, continuaba sin resolverse, de manera que el $15 \mathrm{de}$ febrero se trató de realizar una modificación en el nuevo ordenamiento proyectado, con el fin de llegar a un consenso sobre su aplicación. Efectivamente, se cambió el punto relativo a la obligatoriedad de mantener caballo y armas para acceder a las regidurías, por la obligatoriedad de tener armas o caballo y no desempeñar oficios "vaxos ny viles" ${ }^{39}$. Desconocemos que ocurrió en los años inmediatamente posteriores, si bien, los Libros de actas concejiles (conservados a partir del año 1504), ponen de manifiesto que el sistema electoral tradicional estaba vigente a inicios del siglo $X V I^{40}$. La reforma electoral, en consecuencia, o no se aplicó definitivamente o tuvo un corto período de vigencia en el mejor de los casos.

No fue desde luego el de Calahorra el único ejemplo conocido en el territorio riojano, donde sabemos que en la ciudad de Santo Domingo de la Calzada se produjeron por estas mismas fechas debates similares por la introducción de un nuevo ordenamiento electoral, que finalmente tampoco fue aplicado por la oposición de un sector de la sociedad política local de la ciudad calceatense ${ }^{41}$. Como hemos estudiado en otro lugar, los proyectos de reforma de los sistemas municipales tradicionales fueron temas controvertidos y conflictivos en La Rioja, ya que su aplicación tropezaba en muchos casos con unas estructuras organizativas y de reparto del poder firmemente asentadas en las ciudades, por lo menos desde la época plenomedieval ${ }^{42}$. Por lo que respecta a Calahorra, las principales innovaciones aplicadas durante el reinado de los Reyes Católicos, se plasmaron en el establecimiento al frente del gobierno urbano de un corregidor permanente y de su teniente, y en otras dos medidas a las que todavía no hemos hecho referencia: creación del Ayuntamiento e introducción de un nuevo oficial en el organigrama de cargos municipales dotados de poder político. Me refiero al diputado, magistrado que en teoría debía representar al vecindario de la ciudad, pero que en la práctica se convirtió en un cargo controlado y monopolizado por la oligarquía, pues era elegido de forma directa por los mismos miembros del Regimiento ${ }^{43}$. Las diputaciones desplazaron, por lo tanto, a las anteriores

A.G.S.: R.G.S., fol. $180(14-11-1498)$

A.G.S.: R.G.S., fol. 181 (15-11-1498)

* A.M.C.: L.A. 1504, sesión de 18 de febrero

"FCO. Javier GoicoleA JuLiAN, "Sociedad y relaciones de Doder en una ciudad riojana a fines del Medievo: Santo Domingo de la Calzada..., o.c., pp. 243-286.

* Fco. Javier Goicolea JULIAN, "Concejos urbanos en La Rioja Alta a fines del Medievo: Aspectos institucionales y políticos". Historia. Instituciones. Documentos. $n .26$ (1999), pp. 233-254 y "Sociedad y poder concejil. Una aproximación a la élite dirigente urbana de La Rioja Alta medieval, Studia Historica, H. ${ }^{3}$ Medieval, n. .17 (1999), pp. 87-112.

4: Eran 8 los siputados de Calahorra. En 1505 ejercieron este cargo Diego Álvarez bachiller, Juan Guerrero, Antón Gualite, Gonzalo Muñoz, Diego de Tejada, Martín Fernández Ballestero, Juan del Caño y Juan Martinez Bambilla el mozo (A.M.C.: L.A. 1505) 
fórmulas de representación política vecinal en las sesiones concejiles, lo que abrió el camino al cierre definitivo de las mismas en el nuevo Ayuntamiento. La medida significaba la concesión de un nuevo y firme impulso al control oligárquico del poder, que salió reforzado asimismo mediante los requisitos sociales, profesionales y de fortuna estipulados para poder acceder a las diputaciones y a las demás magistraturas principales. Era el inicio de un nuevo modelo de reglamentación municipal encaminado al mismo tiempo a hacer más efectivo y profesional el trabajo de los oficiales de justicia, regidores y diputados en el nuevo Ayuntamiento de Calahorra, tal y como se ponía de manifiesto en la sesión de apertura en 1508: «Que propuesta la causa sobre que se ayuntan, cada uno de los regidores en su grado aya de votar sobre ella e de su voto syn bollizio alguno, e despues de votado los regidores ayan de votar los diputados segund e commo estuvieren asentados por su orden, e que votando cada uno de ellos ayan de estar callando y oyr lo que dixieren, y el que otra forma tuviere en el votar, que no se consienta ${ }^{44}$.

\section{LA SOCIEDAD DE CALAHORRA EN LOS PRIMEROS AÑOS DE LA EDAD MODERNA}

De acuerdo con el recuento vecinal confeccionado en Calahorra en 1512 con objeto de recaudar el impuesto de la Moneda forera, la ciudad contaba en esta fecha con setecientos treinta vecinos. El número de pobladores de Calahorra superaba, por tanto, al de otros núcleos urbanos riojanos como Haro, Santo Domingo de la Calzada, Navarrete o Briones ${ }^{45}$, pero se situaba por debajo de la ciudad riojana más poblada en los años finales de la Edad Media e inicios de la Moderna: Logroño ${ }^{46}$.

En lo referente al ámbito territorial de control urbano, pues Calahorra ejercía un dominio señorial sobre un territorio rural en el que se hallaban integradas las aldeas de Aldeanueva, Murillo y Rincón de Soto, el conjunto de pobladores aldeanos sumaba algo más de trescientos vecinos, siendo la aldea más poblada Aldeanueva, seguida de Rincón de Soto (donde destacaban los exentos, incluido un caballero) y de Murillo, por este orden. El grueso de la población de la ciudad y de su territorio se concentraba, por tanto, en el núcleo urbano.

El padrón de Calahorra nos informa asimismo sobre los vecinos que habian emigrado de la ciudad en los años inmediatamente anteriores a 1512 ,

4 A.M.C.: L.A. 1508, sesión de apertura del Ayuntamiento.

${ }^{45}$ FCo. Javier GolCOLEA JULIAN, Haro: Una villa riojana..., o.c., "Sociedad y relaciones de poder en una ciudad riojana a fines del Medievo: Santo Domingo de la Calzada.., o.c., pp. 243-286, «Navarrete a finales de la Edad Media: Gobierno y sociedad en una villa riojana de señorio", Berceo, n. ${ }^{9} 136$ (1999), pp. 59-86. los datos sobre Briones pueden consultarse en (A.R.CH.V.: PROTOCOLOS, C. 137-6).

4t José Ramón Diaz de Durana y Ernesto Garcia Fernandez, Demografía y sociedad: La población de Logroño a mediados del siglo XV, Logroño, 1991. 
en total 31 vecinos residentes en las collaciones de Media villa y San Andrés, así como sobre la distribución de la población en el interior del recinto urbano. Efectivamente, los pobladores de Calahorra se distribuían en tres collaciones, de las cuales la más poblada era la collación de Santiago, seguida de las collaciones de Media Villa y San Andrés.

Desde un punto de vista social, lo primero que llama la atención en el núcleo urbano es el importante número de vecinos exentos, un $46 \%$ del total, frente al $54 \%$ que aparecen inscritos como cuantiosos o pecheros. En este sentido, el número de exentos de Calahorra a inicios del siglo XVI, superaba en proporción al número de exentos que se inscribian en padrones confeccionados por estas mismas fechas en otros núcleos urbanos riojanos como Haro o Navarrete ${ }^{47}$, aunque, por el contrario, era menor que en Logroño según los datos que nos proporciona un padrón de la Moneda forera de esta ciudad a mediados del siglo $X V^{48}$. Entre los exentos que se inscribían en Calahorra se encontraban pobres, clérigos, familiares de la Inquisición, varios oficiales y profesionales, e incluso un estudiante; pero, sin duda, el grueso de la población exenta estaba compuesto por la nobleza laica (hombres de armas, caballeros, hidalgos, dueñas, etc.), que tenian su residencia en las diferentes collaciones de la ciudad.

Si nos atenemos a la distribución social en cada una de las collaciones del núcleo urbano, podemos comprobar que la situación era similar en las collaciones de Santiago y de Media villa: un $51 \%$ de pecheros frente a un $49 \%$ de exentos en el primer caso, y un $53 \%$ de pecheros frente a un $47 \%$ de exentos en el segundo caso. La proporción de pecheros era, sin embargo, bastante mayor en la collación de San Andrés, donde el porcentaje se elevaba al $72 \%$, frente a un $28 \%$ de exentos.

Dentro del grupo de los exentos, como ya hemos dicho, destacaban los representantes de la nobleza laica. Un sector nobiliario que no conformaba un grupo social homogéneo, pues los propios empadronadores diferenciaban a los caballeros e hidalgos notorios cuya condición nobiliaria estaba fuera de toda duda, de aquellos vecinos cuya nobleza no estaban clara o no había sido suficientemente probada. Los primeros, menos numerosos, representaban el $22 \%$ de los exentos de la ciudad, mientras los segundos, bastante más numerosos, representaban nada menos que el $47 \%$ del conjunto de los exentos; cifras a partir de las cuales podemos deducir que en la Calahorra de fines de la Edad Media, como también estaba ocurriendo en otros núcleos urbanos riojanos por estas fechas ${ }^{49}$, había un creciente inte-

\footnotetext{
4. FCo. Javier GoICOLEA JULIAN, Haro. Una villa riojana..., o.c., p. 36, "Navarrete a finales de la Edad Media..., O.C., pp. 59-86.

4s José Ramón Diaz de Dupana y Ernesto Garcia Fernandez, Demografía y sociedad..., o.c., p. 25

${ }^{49}$ FCo. Javier Goicolea Julián, Haro. Una villa riojana..., o.c., p. 39-42, "Navarrete a finales de la Edad Media..., o.c., pp. 59-86, y «Sociedad y relaciones de poder..., o.c., pp. 243-286.
} 
rés de determinados sectores pecheros urbanos por acceder a la hidalguía y consolidarse dentro del estado noble de la ciudad.

Una de las fórmulas que tenían estos vecinos para ser considerados exentos era el mantenimiento de armas y caballos, lo cual les permitía desempeñar una función militar en la ciudad como caballeros armados. De esta manera se equiparaban en cierta medida a la nobleza, y el mantenimiento de armas y caballos les podía facilitar, además, la obtención de una sentencia de hidalguía favorable; de ahí el interés manifestado por un buen número de vecinos en demostrar su pertenencia al estado noble, apelando para ello a su condición de caballeros armados con objeto de inscribirse como hidalgos en los padrones $y$, por tanto, no contribuyentes «en pedidos e monedas e moneda forera $e$ otros pechos e tributos e derramas $" 50$.

De la misma forma, a través de los listados de los vecinos de Calahorra que firman con los Reyes Católicos contratos de acostamiento para el mantenimiento de caballo y armas, podemos constatar también la presencia de vecinos "que dizen ser hidalgos", junto a algún pechero acomodado como el sastre Pedro de Arteaga ${ }^{51}$. En definitiva, razones económicas, de prestigio e imagen de poder hacian del acceso a la hidalguía un objetivo muy codiciado; más aun cuando, como ya sabemos, las disposiciones enviadas a la ciudad por los Reyes Católicos en los años finales del siglo XV, tendían a reservar las principales magistraturas urbanas a aquellos vecinos que poseían caballo y armas.

La integración de algunos de estos vecinos en el estado hidalgo y su equiparación a la nobleza antigua, tropezó con la oposición, por un lado, del gobierno municipal, representado mayoritariamente por el estado ciudadano; $y$, por otro lado, con la propia oposición de los sectores de la nobleza notoria. que por lo menos en un primer momento vieron con recelo la llegada de advenedizos a su estado y al organismo que les representaba: la cofradía de caballeros-hidalgos. Efectivamente, a partir de un documento del año 1479, podemos comprobar cómo el gobierno municipal de Calahorra se negaba a considerar como pertenecientes al estado noble, a varios vecinos, entre ellos un carnicero y un herrero, que afirmaban haber sido recompensados con privilegios "de essençion e ffidalguia» por el monarca Enrique IV ${ }^{52}$. Esta actitud era apoyada en estos momentos por los hidalgos notorios, pues ambos sectores temian que el incremento de los vecinos exentos rompiera el acuerdo fiscal al que se había llegado en la ciudad, según el cual en los repartimientos efectuados para "costas comunes", los hidalgos debían pagar "del millar çiento e çinco maravedis y lo restante los otros vesinos de la çibdad "53.

A.G.S.: R.G.S., fol. $80(13-||-1480)$ y fol. $81(13-|| 1-1480)$

"Entre los que "dezian ser hidalgos" se encontraban Martín de Arnedo, Rodrigo Hurtado o Juan Pérez de Vergara (A.G.S.: CONTADURIA DEL SUELDO, $1 .^{a}$ serie, leg. 7).

A.G.S. CAMARA DE CASTILLA, Pueblos, leg. 5.

Ibidem. 
En 1497 contamos con nuevas referencias a estas cuestiones. En efecto, en este año el cabildo de los caballeros hidalgos había requerido al poder real, para que interviniera con objeto de anular un mandamiento del corregidor, Francisco Pérez de Vargas. En este mandamiento, el corregidor ordenaba empadronar como pecheros a todos los hidalgos que no fueran notorios de la dieta, a la vez que instaba a los miembros de la cofradía noble a que no aceptaran en esta institución a ningún hidalgo que no tuviera sentencia de corte. Podemos comprobar, por lo tanto, que la actitud del estado noble era ahora distinta, favoreciendo la integración de los hidalgos de privilegio en la cofradia, muy probablemente con la intención de que se fortaleciera cuantitativamente su estado. Por el contrario, ni al corregidor ni al estado ciudadano les interesaba que aumentara el número de exentos de la ciudad, pues de esta manera se reducía la capacidad contributiva de Calahorra y, por consiguiente, debería incrementarse también la carga fiscal sobre la población pechera. ${ }^{54}$

Los debates por esta cuestión continuaron a inicios del siglo XVI, tal y como nos lo confirma el padrón de la Moneda forera de 1512, en el que los empadronadores diferenciaban a los caballeros e hidalgos de los que "dezian ser hidalgos", aunque todo ellos fueran inscritos en un principio como exentos. Los corregidores y los miembros de la sociedad política local de Calahorra, especialmente los representantes del estado ciudadano, no cejaron en su empeño por controlar y fiscalizar al máximo que los ascensos sociales al estado hidalgo cumplieran todos los requisitos legales y formales, más aun si tenemos en cuenta que la población pechera de Calahorra apenas creció entre 1512 y 1524. De esta forma, si en los datos del padrón de 1512 la población pechera del núcleo urbano ascendía a cuatrocientos vecinos, en la sesión de ayuntamiento del 21 de enero de 1524 se seguía constatando que "en la çibdad hay unos cuatroçientos pecherns o pocos mas" $" 55$.

Dentro del grupo de los exentos destacaban también los clérigos, pues debemos tener en cuenta que nos encontramos en una ciudad sede de obispado. En total se inscribían setenta y cuatro clérigos en el padrón, la mayor parte de los cuales tenían su residencia en las collaciones de Media Villa y de Santiago, por este orden. Entre los clérigos distinguimos al chantre y al sochantre, al deán y al arcediano de Berberiego, así como al licenciado de la gramática, que se ocupaba de enseñar a leer y a escribir a los niños y jóvenes de las familias más acomodadas de la ciudad. También estaban exentos los familiares del cabildo, el alguacil del obispo, un estudiante y algunos profesionales, como el médico doctor de Yze, o el platero maestre Hernando, ambos residentes en la collación de Santiago. Por fin, tampoco

\footnotetext{
A.G.S.: R.G.S. (6-V-1497)

5.M.C.: L.A. 1524, sesión del 21 de enero.
} 
nos podemos olvidar de los pobres, que se encontraban repartidos de la siguiente forma en las collaciones: diez en la collación de Santiago, cinco en la de Media Villa y cuatro en la de San Andrés.

Ahora bien, si nos atenemos a los profesionales inscritos en el padrón, podemos comprobar que, salvo unas pocas excepciones, la mayoría pertenecian al grupo de los pecheros o cuantiosos. Estos profesionales se concentraban fundamentalmente en la collación más poblada de la ciudad: la de Santiago, a la que seguían a una distancia considerable las collaciones de Media Villa y San Andrés, respectivamente. Como sucedia en otros núcleos urbanos riojanos, entre los artesanos sobresalían los profesionales dedicados al sector texti ${ }^{56}$, destacando especialmente en el padrón los sastres y los tejedores, aunque también se inscriben un soguero, un pañero y un pelaire. No se hace, sin embargo, referencia a tundidores, aunque sabemos que éstos realizaban su trabajo en la ciudad, pues existía un veedor que supervisaba sus labores. El 21 de julio de 1541 podemos constatar la realización de un ordenamiento por parte del Ayuntamiento de Calahorra, reglamentando las cantidades de dinero que podían percibir los tundidores por su trabajo: "Ansimismo hordenaron que ningund tundidor sea osado de llevar de la vara de Refino por tundir mas de un quartillo so pena de çien maravedis repartidos en la forma susodicha a dos tijeras, y de cada vara de Congay de la tierra con dos tijeras siete maravedis, y de la vara de Frisado de nueve quarteles y de arriba diez maravedis. Yten que en todos pannos refrisen los enveses sin llevar mas por ello de los preçios dichos, $y$ de la vara de veynteno de Segovia seis maravedis...". El ordenamiento continuaba reglamentando el trabajo con otro tipo de paños, como el Pardillo, Bruneta, Cordellate o de Londres ${ }^{57}$.

A los artesanos del sector textil les seguían en número los trabajadores del cuero y de la piel, pellejeros, correros y, sobre todo, zapateros (cinco estaban avecindados en la collación de Santiago). Mientras, entre los profesionales del metal, tercer sector artesanal fundamental que se vislumbra a través del padrón de 1512, destacaban los herreros. La lista de artesanos se completaba con algún astero, yesero, fustero, tornero o carpintero, y con dos plateros avecindados en la collación de Santiago. Estos últimos se encargaban de realizar los pesos utilizados en las tiendas y carnicerias de la ciudad, afinaban los instrumentos de medición, e igualmente elaboraban objetos suntuarios destinados a las iglesias y a los sectores sociales urbanos acomodados.

Entre los profesionales del sector terciario constatamos oficios como carnicero, tendero, hornero, carretero, barbero, criado, maestro, boticario, ciru-

"FCo. Javier Golcolea JuLian, "Mundo urbano y actividades económicas en La Rioja Alta bajomedieval», Espacio, Tiempo y Forma, $H^{a}$ Medieval, n. 11 (1998), pp. 243-283.

A.M.C.: L.A. 1541, sesión del 21 de julio. 
jano o escribano; aunque en todo caso conviene tener presente que, como es habitual en textos de estas características, realizados con fines exclusivamente fiscales y no con la intención de registrar las profesiones de los vecinos, no se recogen en ellos todos los oficios. De ahí que los empadronadores sólo señalen a unos pocos representantes del sector primario, sector que sin embargo ocupaba a un porcentaje muy importante de la población pechera de Calahorra. Con toda probabilidad los empadronadores no consideraban necesario señalar la actividad de los vecinos que trabajaban en las labores agropecuarias, por ser esta actividad la más generalizada y conocida, de manera que únicamente se debían de preocupar por diferenciar en la media de lo posible al resto de profesionales.

Los labradores, pastores, artesanos, tenderos, etc., integrados en su mayoría en el común de pecheros de Calahorra, tenian sus casas junto a las suntuosas mansiones de los miembros de la élite ciudadana e hidalga. En efecto, la collación de Media Villa y, sobre todo, la de Santiago eran los lugares de residencia de los representantes de la oligarquía. En esta última collación vivían la mayor parte de los hidalgos, como el licenciado Liçaor, Diego Sánchez de Tejada o Hernando de Bobadilla (dudoso), caballeros como Pedro de Fontano, o ciudadanos como Francisco de Yanguas y Antón Gualite. Todos ellos poseían los niveles de renta más elevados de la ciudad, y a través de los Libros de actas municipales podemos comprobar asimismo que tuvieron una activa participación en el gobierno municipal de Calahorra entre 1504 y 1520. El licenciado Liçaor fue diputado en 1512, regidor en 1513 y 1515, y teniente de corregidor en 1516; Diego Sánchez de Tejada fue regidor en 1510 y 1520; Hernando de Bobadilla fue diputado en 1509, 1511, 1514, 1517 y 1520; Francisco de Yanguas fue diputado en 1509 y 1511, y regidor en 1513 y 1519; y, por fin, Antón Gualite fue diputado en 1505 y 1515 , y regidor en $1506,1510,1516$ y $1518^{58}$.

\section{A MODO DE CONCLUSIÓN.}

El pasado medieval de la ciudad riojana de Calahorra presenta todavía bastantes interrogantes, muchos de ellos de difícil resolución por las carencias documentales actuales. Ahora bien, la consulta de documentos de diversas instituciones ha permitido, por lo menos, vislumbrar algunos aspectos de la organización concejil y de la articulación social en este importante núcleo urbano de La Rioja Baja. De esta forma, hemos podido ir viendo cómo el gobierno municipal de Calahorra se fue fortaleciendo a lo largo de los siglos XIII y XIV, a la vez que iba experimentando transformaciones; entre ellas, las derivadas de la introducción del oficio de regidor hacia mediados de la centuria del cuatrocientos, como consecuencia de la reforma municipal establecida durante reinado de Alfonso XI. Ahora bien, no

${ }^{54}$ A.M.C.: L.A. $1504-1520$. 
es menos cierto que las reformas tuvieron que adaptarse a la realidad socioinstitucional de la urbe, de ahí que no todas ellas tuvieran los efectos deseados. Ciertamente, el carácter abierto del concejo calagurritano se mantuvo en la baja Edad Media, al igual que el destacable papel político desempeñado por las collaciones en su condición de células básicas de organización comunitaria del vecindario. Hubo que esperar, por lo tanto, al gobierno de los Reyes Católicos para que los corregidores y sus tenientes desplazaran de sus funciones a los alcaldes ordinarios en Calahorra, y tuviera lugar asimismo el cierre efectivo del concejo con la consiguiente introducción de los diputados en el gobierno municipal. Surgió de esta forma el nuevo Ayuntamiento que regirá la ciudad de Calahorra hasta la introducción de los regimientos perpetuos hacia mediados del siglo XVI.

Lo cierto es que los intentos de reformar las instituciones y los mecanismos de funcionamiento del gobierno municipal, entre ellos el sistema de elección de oficiales (fines del siglo XV), tropezaron muchas veces con la firme oposición de sectores importantes de la sociedad política de Calahorra. $Y$ aqui debemos hacer referencia a la élite dirigente que controlaba el poder municipal en la plena Edad Media, y que varios siglos después seguía dominando los más importantes resortes de poder en el municipio. Eran fundamentalmente familias de comerciantes y hombres de negocio integrados en el estado ciudadano, que a lo largo de la baja Edad Media habían ido perdiendo cotas de poder frente a la nobleza urbana, pero que todavía a finales del siglo XV e inicios del XVI seguian teniendo una representación mayoritaria en el gobierno municipal. Frente a los ciudadanos, los caballeros e hidalgos de Calahorra, integrados en su propio cabildo, habían ido adquiriendo una influencia política creciente en la ciudad, paralela a su aumento en número por el avecindamiento de caballeros e hidalgos notorios, y por el incremento en la urbe de los vecinos cuantiosos que decían contar con hidalguías de privilegio. En efecto, en el otoño del Medievo, el acceso a la nobleza como fórmula para lograr exenciones fiscales, pero sobre todo como requisito indispensable y decisivo en el ascenso social en el medio urbano, motivó que un número importante de vecinos de Calahorra trataran de acceder a la hidalguía, tal y como se pone de manifiesto en el padrón de 1512. El mantenimiento de caballo y armas, acorde con el ideal caballeresco de la época, podía ser un requisito para alcanzar tan ansiado estado; si bien, de la documentación conservada se deduce que la asimilación de hidalgo dudoso a noble notorio, no era un objetivo fácil de lograr, sobre todo si no se poseía el nivel de riqueza e influencia adecuados. Por último, frente a la élite noble y ciudadana, el sector social urbano que sufría las consecuencias del ejercicio del poder oligárquico, apenas se deja entrever en la documentación; aunque, desde luego, el común de hombres buenos pecheros de Calahorra, a través de sus procuradores, también participó en la medida de sus posibilidades en la vida política municipal de fines del siglo XV, denunciando las arbitrariedades e irregularidades cometidas por los oficiales concejiles, ante la autoridad real. 


\section{ANEX()}

\section{TABLA I}

DSTRIBUCION SOCIALEN LA CIUDAD DE CALAH TORA Y FN SUS ALDEAS SEGÚN ELPADRON DE LA MONLDA PORERA DE 1.512

\begin{tabular}{|c|c|c|c|c|}
\hline CIUDAD & $\begin{array}{l}\text { Collación de } \\
\text { San Andrés }\end{array}$ & $\begin{array}{l}\text { Collación de } \\
\text { Media Villa }\end{array}$ & $\begin{array}{c}\text { Collación de } \\
\text { Santiago }\end{array}$ & Total vecinos \\
\hline Comonosos & 97 & 130 & 173 & 400 \\
\hline tionges notoros & -... & 3 & 29 & 32 \\
\hline Conoleros & 1 & 2 & 2 & 5 \\
\hline Hombes dramas & & & 1 & $!$ \\
\hline Escudtos & 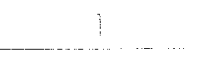 & - & & $\vdots$ \\
\hline Duenis & 2 & 12 & 15 & 29 \\
\hline Congo & 9 & 39 & 26 & 14 \\
\hline Hioduge didesos & 20 & 47 & 1 & 138 \\
\hline Drenosedidesas & $\ldots$ & 3 & 7 & 10 \\
\hline Petes & 4 & 5 & 10 & 19 \\
\hline Fstudiones & 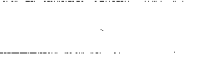 & 1 & - & 1 \\
\hline Fonitiones & - & $?$ & $?$ & 4 \\
\hline $\begin{array}{l}\text { Algunites } \\
\text { donopes }\end{array}$ & $\therefore$ & - & 1 & 1 \\
\hline Othos axom & 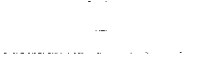 & 3 & 12 & 15 \\
\hline Totol vecinos & 134 & 247 & 349 & 730 \\
\hline
\end{tabular}

\begin{tabular}{|c|c|c|c|c|}
\hline ALDEAS & Aldeanueva & Murillo & Rincón de Soto & Total vecinos \\
\hline Cucultionso: & $13 !$ & 77 & 16 & 254 \\
\hline Hidalgus noturios & - & & 2 & 2 \\
\hline Coballers & - & - & 1 & 1 \\
\hline Duenos & - & 1 & 10 & 11 \\
\hline Clérigos & 4 & 2 & 2 & 8 \\
\hline Hidelgos dudusos & 3 & 5 & 27 & 35 \\
\hline Pobres & - & 1 & 3 & $A$ \\
\hline Total vecilios & 138 & 80 & 91 & 315 \\
\hline
\end{tabular}


TABLA II

PROFESIONALES INSCRITOS EN EL PADRÓN DE 1512

\begin{tabular}{|c|c|c|c|c|c|}
\hline COLLACIONES & N.․ VECINOS & $\begin{array}{c}\text { SECTOR } \\
\text { PRIMARIO }\end{array}$ & $\begin{array}{c}\text { SECTOR } \\
\text { SECUNDARIO }\end{array}$ & $\begin{array}{c}\text { SECTOOR } \\
\text { TERCIARIO }\end{array}$ & $\begin{array}{c}\text { N.․ } \\
\text { PROFESIONES }\end{array}$ \\
\hline SAN ANDRES & 134 & & $\begin{array}{l}\text { SASTRE } 1 \\
\text { IEJEDOR I } \\
\text { ZAPATERO I } \\
\text { PELLEJERO I } \\
\text { CORRERO I } \\
\text { HERRERO ! } \\
\text { ASTERO I }\end{array}$ & $\begin{array}{l}\text { HORNERO } 1 \\
\text { CARNICERO } 1 \\
\text { CARRETERO } 2 \\
\text { DOCTOR } 1\end{array}$ & 12 \\
\hline MEDIA VILLA & 247 & $\begin{array}{l}\text { HORTELANO I } \\
\text { MOLINERO I } \\
\text { CABRERO I } \\
\text { PAIOMERO I }\end{array}$ & $\begin{array}{l}\text { SASTRE } 1 \\
\text { TEJEDOR } 5 \\
\text { PELAIRE I } \\
\text { SOGUERO I } \\
\text { PELIEIERO } 1 \\
\text { HERRERO } 3 \\
\text { FUSTERO } 1 \\
\text { YESERO } 1\end{array}$ & $\begin{array}{l}\text { CRIADO } 1 \\
\text { BARBERO I } \\
\text { MAESTRO I }\end{array}$ & 21 \\
\hline SANTIAGO & 349 & $\begin{array}{l}\text { MOLINERO } 2 \\
\text { PASTOR } 1 \\
\text { BOYERIZOI }\end{array}$ & $\begin{array}{l}\text { SASTRE } 8 \\
\text { TEJEDOR } 3 \\
\text { PANERO } 1 \\
\text { SOGUERO } 1 \\
\text { CINTERO I } \\
\text { ZAPATERO } 5 \\
\text { HERRERO } 2 \\
\text { BALLESTERO I } \\
\text { TORNERO } 1 \\
\text { PLATERO } 2\end{array}$ & $\begin{array}{l}\text { CARNICERO I } \\
\text { TENDERO I } \\
\text { HORNERO I } \\
\text { CARRETERO I } \\
\text { BARBERO ! } \\
\text { BOTKARIO } 1 \\
\text { CIRUJANO I } \\
\text { DOCTOREN } \\
\text { MEDICINA I } \\
\text { DOCTOR I } \\
\text { IICENCIADO } 2 \\
\text { BACHILIER ? } \\
\text { ESCRIBANO I } \\
\text { LICENCIADO } \\
\text { DE LA } \\
\text { GRAMATICA I } \\
\text { TAMBORINO I } \\
\text { PREGONERO } 2\end{array}$ & 47 \\
\hline TOTAL & 730 & 8 & 46 & 26 & 80 \\
\hline
\end{tabular}




\section{GRÁFICO 1. Distribución de la población en la ciudad y aldeas en $\mathbf{1 5 1 2}$}

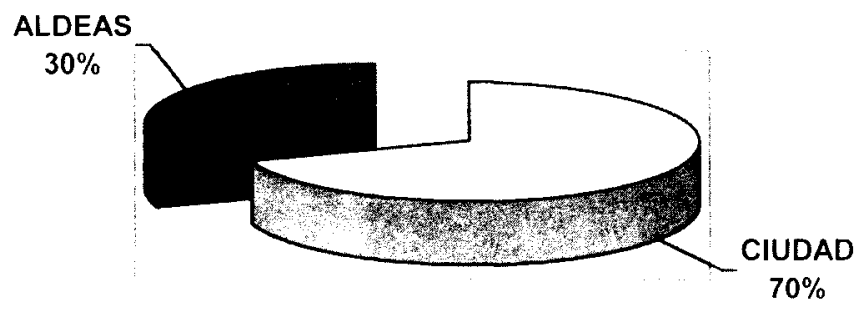

GRÁFICO 2. Distribución de la población en la ciudad en 1512

C. SANTIAGO $48 \%$

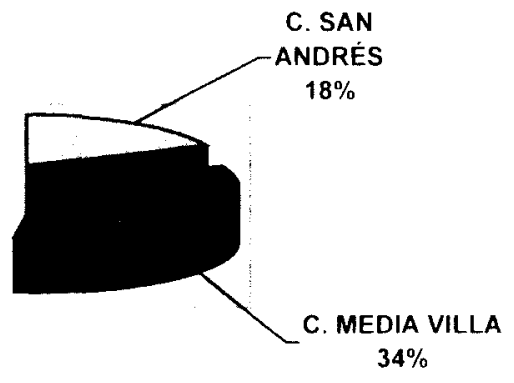

GRÁFICO 3. Distribución de la población en las aldeas en 1512

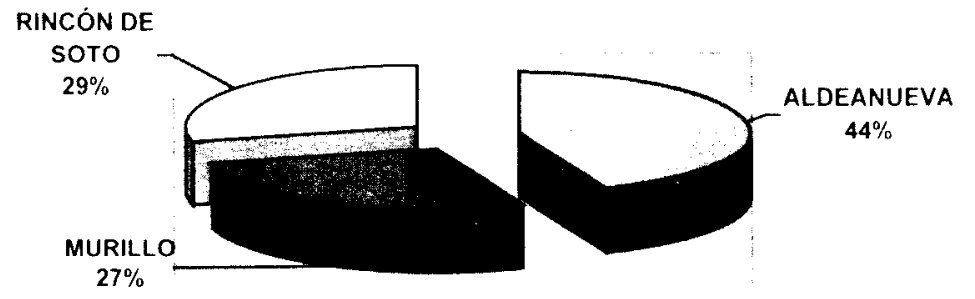




\section{GRÁFICO 4. Distribución social en la ciudad en 1512}

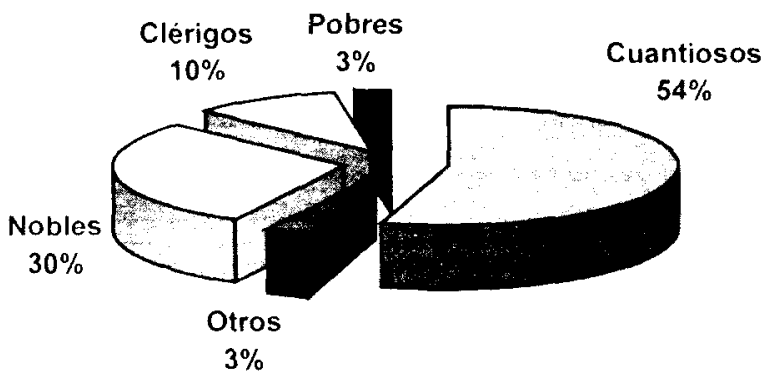

GRÁFICO 5. Distribución social de los exentos en la ciudad en 1512

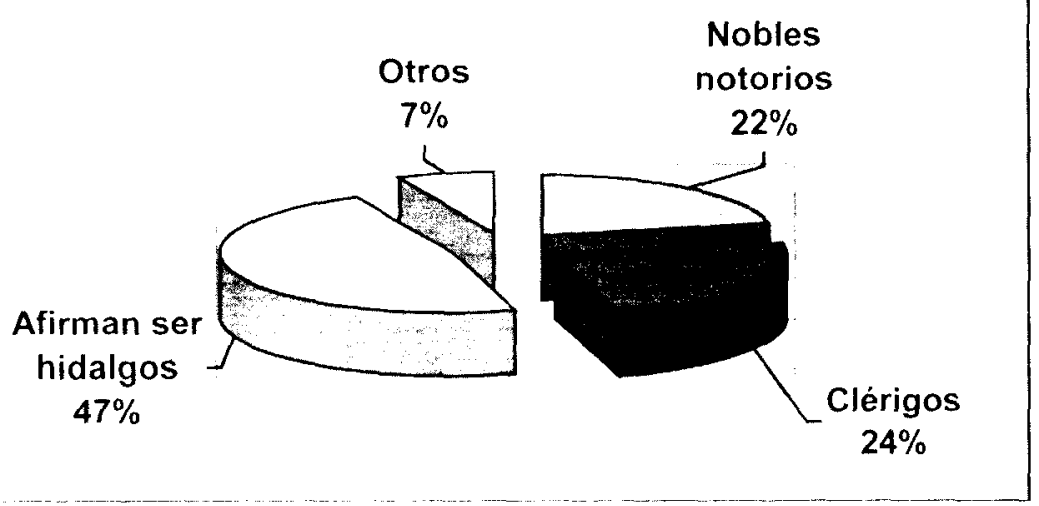



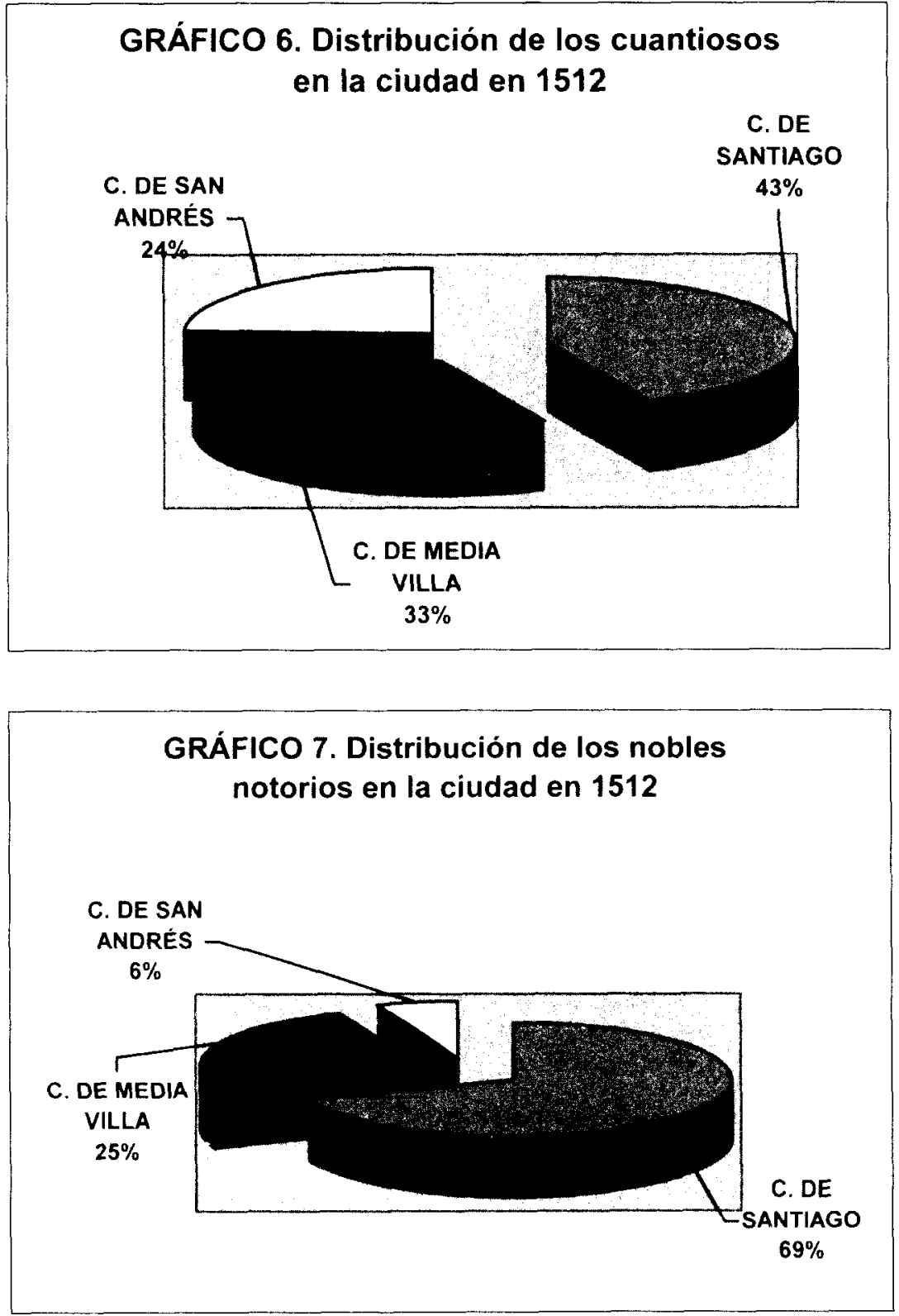


\section{GRÁFICO 8. Distribución de los nobles dudosos en la ciudad en 1512}

\section{DE SAN}

ANDRÉS

$14 \%$

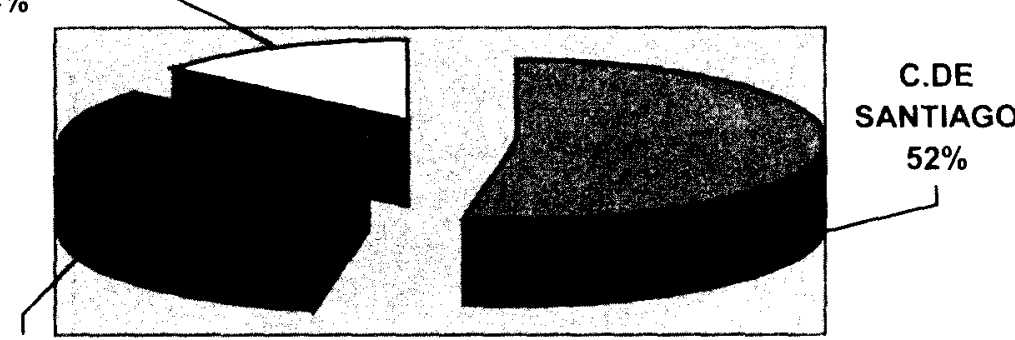

C. DE MEDIA

VILLA

$34 \%$

GRÁFICO 9. Distribución de los clérigos en la ciudad en 1512

C. SAN ANDRÉS

$12 \%$

C. DE MEDIA

VILLA

$53 \%$

ᄂ

C. DE SANTIAGO

$35 \%$ 


\section{GRÁFICO 10. Distribución de los pobres en la ciudad en 1512}
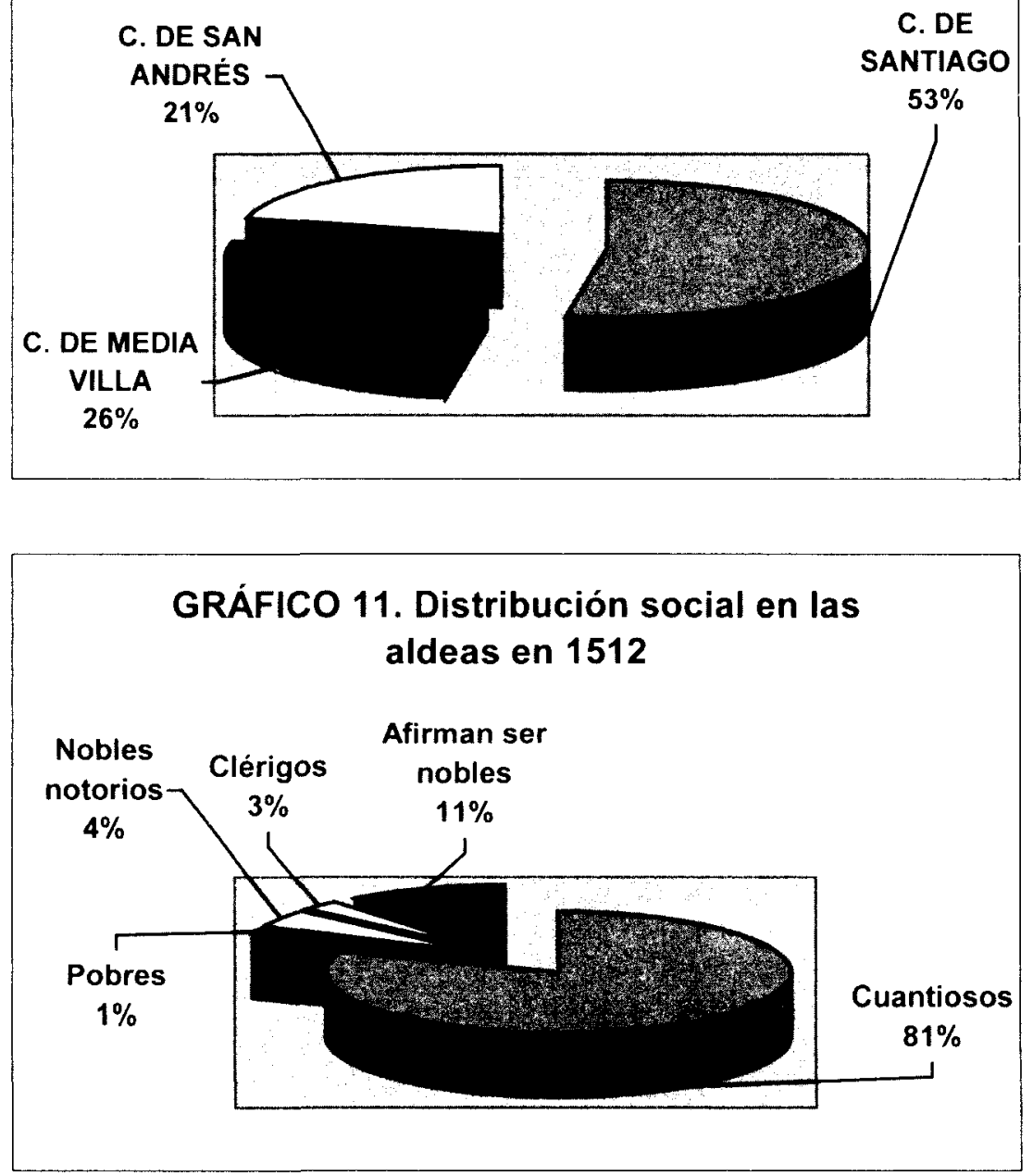

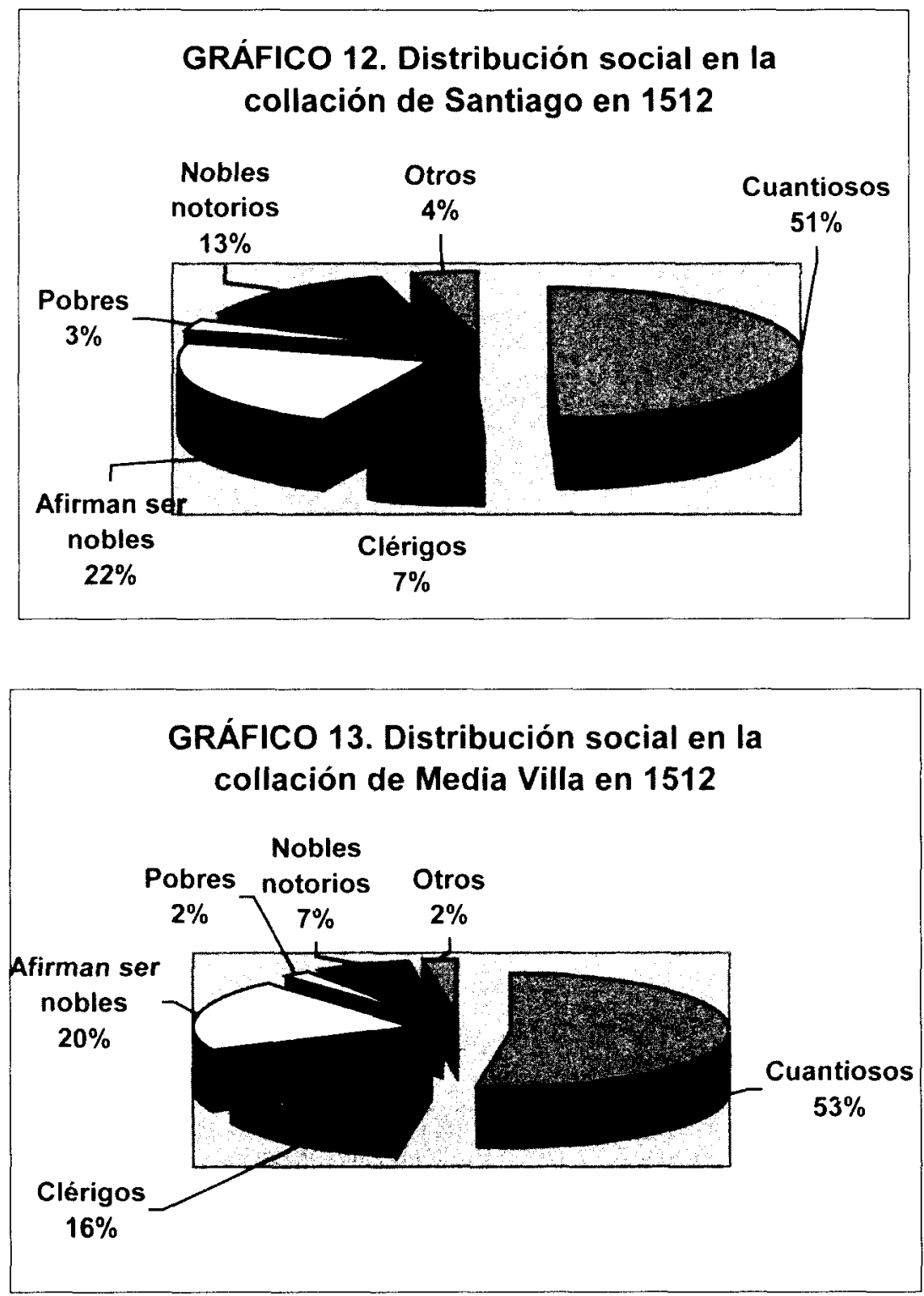


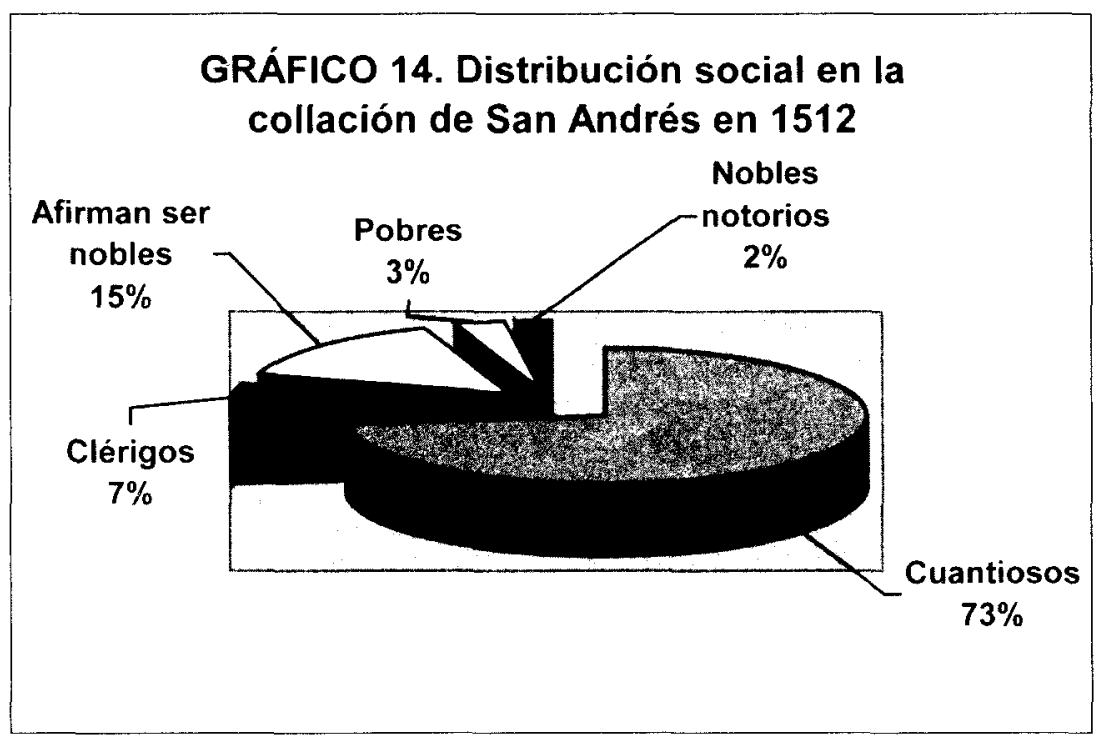

\section{GRÁFICO 15. Distribución social en la aldea de Aldeanueva en 1512}

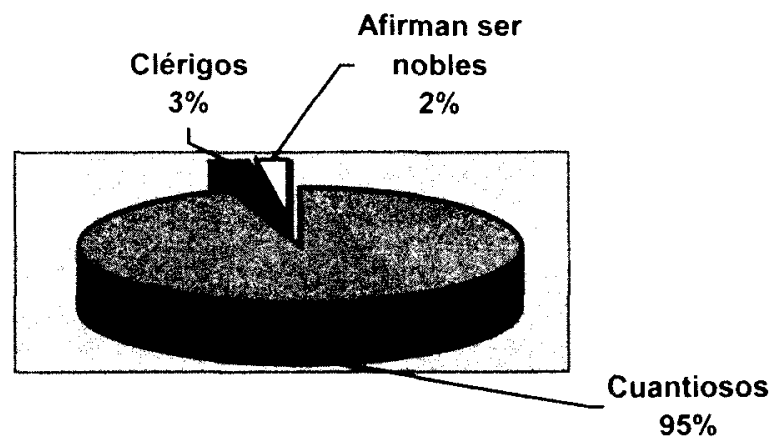



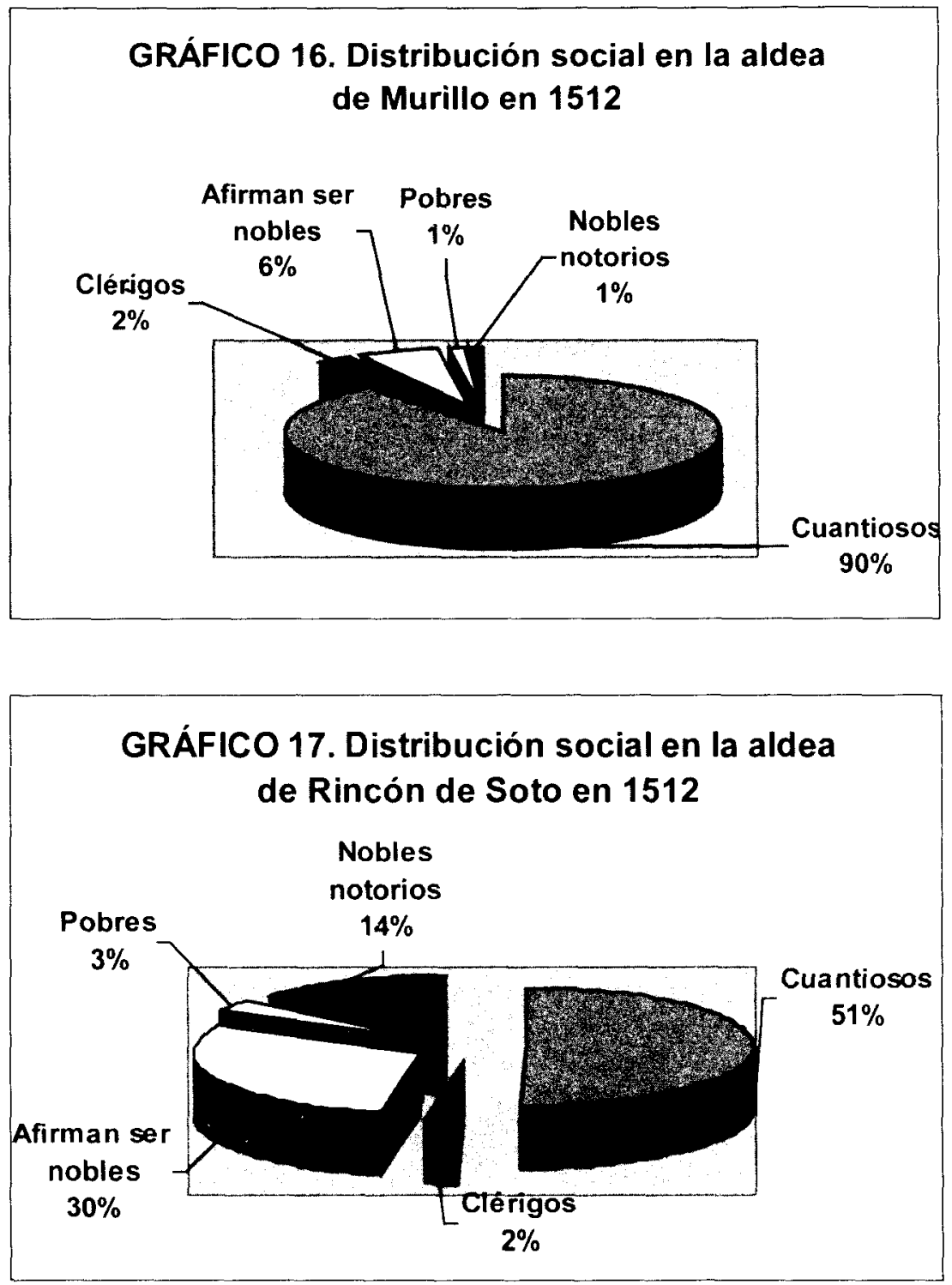


\section{PADRÓN REALIZADO POR EL CONCEJO DE CALAHORRA EN EL AÑO 1512 \\ PARA RECAUDAR EL IMPUESTO DE LA MONEDA FORERA EN LA CIUDAD Y EN SUS ALDEAS}

Fuente A.R.CH.V. Sala de Hijosdalgo, leg. 133, exp. 9

\section{Collación de SAN ANDRÉS}

Martin de Bobadilla

Pedro Diaz

Vernes de Haso

Beltran

Sebastian Gomez

Juan Diaz

Pero Nabarro

Diego Gil

Martin Cabero

Gil Garçes

La Munnoza

Su hermano

Gonçalo Diaz

Miguel San Martin

Diego Gomez

Alonso Martyn

Pero Miguel

Diego Casota

Herederos de Martin

Aguado

El dotor de Molina

Pedro de Ayasa

Juan de Robles

Juan

Juana la Navarra

Miguel Anton

Hernan Tomas

Ruy Garçia Tomas

Pero Moreno

Hernan Martinez

Pero Ximenez

Juan de Aragor

Pedro del Rey

La vieja de Diego

Pedro el astero

Juan Sanches

\section{ydo}

quantioso

dize ser hidalgo

quantyoso

quarityoso

quantyoso

quantyoso

quantyoso

quontyoso

escudero

duena

dize ser hidalgo

dize ser hidalgo

dize ser hidalgo

dize ser hidalgo

clerigo

quantyoso

ydo

quantioso

dize ser hidalgo

dize ser hidalgo

quantyoso

caballero

pobre

pobre

quantyoso

quantyoso

quantyoso

quantyoso

clerigo

quantyoso

quantyoso

quantyoso

quantyoso

quantyoso
Pedro de Salamanca

Martyn de Motrico

Gonçalo Sanz

Falcon

Su cunnada

Diego

Diego de Gonçalo

Pero Ruiz

Pero Ruiz de Corres

Diego Ruyz Vesino

Martyn Abad

Juan Gomez de

Aguisa

Françisco Garçia

Françisco Albierma

Hernan Ruyz

Diego Ruyz

Pero Martinez

Juan de Laguardia

Hernan Feliz

Pero Feliz

Anton de Rabanera

Sancho

Pero Garçia su

hermano

La madre

Martyn herrero

Myguel Ruiz

Sebastian

Juan Garçia su hijo

Juan Ruiz çapatero

Pero Perez

Juan Gimenez

Su hiemo

Markyn pellegero

Martyn Vesino

Pedro Laguna

Juan Calama

Martyn hornero quantyoso

dize ser hidalgo

quantyoso

quantyoso

clerigo

quantyoso

dize ser hidalgo

quantyoso

quantyoso

clerigo

dize ser hidalgo

quantyoso

quantyoso

quantyoso

quantyoso

quantyoso

quantyoso

quantyoso

quantyoso

dize ser hidalgo

clerigo

quantyoso

quantyoso

quantyoso

quantyoso

ydo

ydo

quantyoso

quantyoso

quantyoso

quantyoso

quantyoso

quantyoso

quantioso

ydo

quantyoso 


\begin{tabular}{|c|c|c|c|}
\hline Su hijo & quantyoso & Los de Diego & \\
\hline Pero Martynez & ydo & Rodrigues & quantyoso \\
\hline Gorçio de Martyn & & Pero Ramirez & ydo \\
\hline Sanz & ydo & Diego Bendino & quantyoso \\
\hline Martyn de Zerio & quantioso & Juan de ley & quantyoso \\
\hline Juan Hijano & quantyoso & Juan Martinez & quantyoso \\
\hline $\begin{array}{l}\text { Hernan Gil } \\
\text { Los herederos de }\end{array}$ & clerigo & $\begin{array}{l}\text { Martyn de la Cuesta } \\
\text { Gonçalo Martinez de }\end{array}$ & quantyoso \\
\hline Diego Garçia & quantyoso & Lasesto & quantyoso \\
\hline Martyn Ybonez & dize ser hidalgo & El hierno de Lezana & quantyoso \\
\hline Lope Hernandez & clerigo & Rodrigo de Sojo & quantyoso \\
\hline $\begin{array}{l}\text { Pero Fernandez } \\
\text { La de Myguel }\end{array}$ & quantyoso & $\begin{array}{l}\text { Hernan Crespo } \\
\text { Juan Gomez }\end{array}$ & quantyoso \\
\hline $\begin{array}{l}\text { Serrano } \\
\text { Francisco Castro }\end{array}$ & duena & $\begin{array}{l}\text { carretero } \\
\text { Pero Diez de }\end{array}$ & quantyoso \\
\hline sastre & quantioso & Montemayor & quantioso \\
\hline Sebastion hierno de & & Hurraca & pobre \\
\hline Martyn Ybanez & quantyoso & Pero Feliz & quantyoso \\
\hline $\begin{array}{l}\text { Myguel Loreynte } \\
\text { Juan Perez e Diego }\end{array}$ & quantyoso & $\begin{array}{l}\text { Gil Jordan } \\
\text { Gonçalo Ximenez }\end{array}$ & $\begin{array}{l}\text { quantyoso } \\
\text { clerigo }\end{array}$ \\
\hline Perez & quantyoso & Diego Martinez & dize ser hidalgo \\
\hline Juan Laguna & quantyoso & Buytrago & quantyoso \\
\hline Martyn Garcia & & Pedro de Sangre & quantyoso \\
\hline correro & quantyoso & Hernan Gutierrez & quantyoso \\
\hline Juan Fernandez & quantyoso & Hernando Gascon & dize ser hidalgo \\
\hline Hernando de & & Ei hierno de Luys & \\
\hline Horgandona & dize ser hidalgo & Talynero & quantioso \\
\hline Martyn Sanz & & Juan Calbo & quantioso \\
\hline carnizero & quantyoso & Martyn de Ayala & dize ser hidolgo \\
\hline Gil Fernondez & quantioso & Alonso de la Torre & dize ser hidalgo \\
\hline Juan de Alfaro & quantyoso & Sancho Sanchez & quantyoso \\
\hline Juan Garçia Hayo & quantyoso & La de Diego & \\
\hline Pedro Balnotera & quantyoso & Martinez & quantyoso \\
\hline El hijo del Burgo & clerigo & Sebastian su sobrino & quantioso \\
\hline Juan de Monylla & quantyoso & Juan Moreno & quantioso \\
\hline Cotalino Hernandez & quantyoso & Anton tejedor & quantyoso \\
\hline Pero Navarro & quantyoso & Juan Garçia de & \\
\hline Juan Guerrero & quantyoso & Arancon & quantioso \\
\hline Pedro de Monilla & pobre & Gil Gutierrez & quantyoso \\
\hline Juan Sanz Roldan & dize ser hidalgo & Diego Domingo & quantyoso \\
\hline Diego Mangado & quantyoso & Pedro de Meryno & dize ser hidalgo \\
\hline Hernan Cabero & dize ser hidalgo & Juan Martinez & \\
\hline Gonçalo de Garçia & & carretero & quantyoso \\
\hline Perez & ydo & Sancho Mano & quantyoso \\
\hline Pero Ruyz Lero & quantyoso & Gonçalo Ruyz & quantyoso \\
\hline
\end{tabular}




\section{Collación de MEDIA VILLA}

Myguel Rubio

Gil Fernandez

Gonçalo Rubio

Juan Redivano

Diego Perez su hijo

Myguel Gascon

Catalyna de Duranda

Su hierno Diego

Gutierres

Juan Ruyz

Hernan Ganyto

Bernal Marçilla

Juan Tomas Escudero

Juan Lorenço

Domyngo Serrano

La de Diego Galosa

Gonçalo Lopes

Gonçalo Ruyz

Pero Felis

Diego Nalda

Myguel Ruiz yesero

Pero Firyno

Diego de Solar

Garçia tejedor

Bernaldino de Arnedo

Maestre Juan

Bartolome de Albas

Pero Perez nieto de

Cornago

Gonçalo Marçilla

Myguel de Cerbenna

Esparça

Garçia cabrero

La de Ferrago

Hernan Gomez

Garcia de la Fuente

Pedio de Arteaga

sastre

Diego el tejedor

Maria de Arze quantyoso

quantyoso

quantyoso

quantyoso

quantyoso

quantyoso

quantioso

ydo

quantyoso

clerigo

quantyoso

estudiante

quantyoso

quantyoso

quantyoso

quantyoso

quantyoso

quantyoso

quantyoso

quantyoso

quantioso

clerigo

quantyoso

quantioso

quantioso

dize ser hidalgo

dize ser hidalgo

clerigo

quantyoso

clerigo

quantyoso

quantyoso

quantyoso

dize ser hidalgo

quantioso

quantioso

pobre
Cereso

Montesino

Juan de Ronçesballes

Françisco de

Ronçesballes

Pero Sanz

El hierno de Juan

Aller

Juan Quesoa

Julian

La de Perucho de

Arteaga

Hernan Sanz de

Lumbreras

Diego Martynez

La de Juan Martynez

Maria de Tordesillas

Ximeno palomero

La de Juan Diaz

pellegero

La de Juan Lopez

Anton tejedor

Juan Moreno

El sochantre

Su madre

Pedro Capata

Bernaldino de

Arellano

Martyn Gutierrez

Su hija

Juan Martynez de

Muro

Juan Gutierrez

Juan Guerrero

Juan de Ybar

Martyn Sanz de

Briviesca

Ynygo de Bribiesca

Morales

Ante: Ruyz

Bastida

Cobaçar

Juan Gutierrez

Casasola

La de Diego de Soria clerigo

quantyoso

dize ser hidalgo

dize ser hidalgo

quantyoso

quantioso

dize ser hidalgo

quantyoso

dize ser duena

quantioso

clerigo

quantyosa

quantyoso

quantioso

quantioso

quantyoso

quantyoso

quantyoso

clerigo

quantyoso

ydo

quantioso

quantyoso

pobre

derigo

quantioso

quantyoso

quantyoso

clerigo

quantyoso

clerigo

quantyoso

clerigo

clerigo

quantyoso

derigo

quantyosa 


\begin{tabular}{|c|c|c|c|}
\hline Andres Gomez & clerigo & Diego Alonso & clerigo \\
\hline Diego Gomez & quantyoso & Françisco de Jubera & clerigo \\
\hline Juan Guerra & dize ser hidalgo & Sebastian & familiar \\
\hline La de Rodrigo de la & & El chantre & clerigo \\
\hline Questo & quantiosa & Bernal Santoro & clerigo \\
\hline Anton Myguel & quantyoso & Pero Alvarez & ydo \\
\hline Rodrigo de Cores & clerigo & Pero Françisco & quantyoso \\
\hline Ynigo de Urtado & clerigo & Juan Martynez de & \\
\hline La de Juan Fernandez & duena & Munilla & quantyoso \\
\hline El ama del chantre & quantyosa & Pero Françisco el & \\
\hline La muger de Onorio & quantiosa & $\begin{array}{l}\text { moço } \\
\text { Leonor de Raedo }\end{array}$ & $\begin{array}{l}\text { quantioso } \\
\text { quantioso }\end{array}$ \\
\hline Rodrigo Navarro & $\begin{array}{l}\text { dize que es } \\
\text { hidalgo }\end{array}$ & $\begin{array}{l}\text { Juan Martynez de } \\
\text { las Heras }\end{array}$ & quantyoso \\
\hline Françisco Guerrero & clerigo & Juan de Sesmo & quantyoso \\
\hline La de Juan Garrido & $\begin{array}{l}\text { dize que es } \\
\text { duena }\end{array}$ & $\begin{array}{l}\text { La otra Catalyna } \\
\text { El hospitalero }\end{array}$ & $\begin{array}{l}\text { dize ser duena } \\
\text { no quantioso }\end{array}$ \\
\hline Elbira Falcon & quantyosa & El maestro Baço & clerigo \\
\hline Su hierno & quantyoso & Diego del Tello & quantyoso \\
\hline Juan Ruyz de & & Su madre & donaçion \\
\hline Tudelilia & $\begin{array}{l}\text { dize que es } \\
\text { hidalgo }\end{array}$ & $\begin{array}{l}\text { Pero Fernandez } \\
\text { Juan Martynez }\end{array}$ & $\begin{array}{l}\text { clerigo } \\
\text { clerigo }\end{array}$ \\
\hline Gonçalo Moreno & quantyoso & Juan Marçilla & quantyoso \\
\hline Pero Fernandez & & El dean & clerigo \\
\hline del Poço & clerigo & Beałriz & quantyoso \\
\hline Gil su criado & quaníyoso & Juan Martynez de & \\
\hline Pero Martinez de & & Gamarra & clerigo \\
\hline Cerbera & dize que es & Mari Gamarra & quantyoso \\
\hline & hidalgo & Garçia Roncal & quantyoso \\
\hline El hijo de Romero & dize que es & Pedro el tejedor & quantyoso \\
\hline & hidalgo & Anton Perez & clerigo \\
\hline Myguel Martynez & clerigo & Bernal Perez & quantioso \\
\hline Nycolas de Salamanca & quantioso & Hernando el fustero & quantioso \\
\hline Juan de Grennon & dize ser hidalgo & Françisco de Sosa & dize ser hidalgo \\
\hline Juan Martynez & clerigo & Juan del Prado & dize ser hidalgo \\
\hline Diego de Ayala & $\begin{array}{l}\text { dize que es } \\
\text { hidalgo }\end{array}$ & $\begin{array}{l}\text { Rodrigo Hurtado } \\
\text { El arçediano }\end{array}$ & dize ser hidalgo \\
\hline Juan de Ayala & clerigo & Berbediego & clerigo \\
\hline Juan de Bicastillo & dize que es & Hernan Gimenez & clerigo \\
\hline & hidalgo & El arzediano de Lorba & clerigo \\
\hline Hernando de Grennon & dize ser hidalgo & Elvintra & clerigo \\
\hline Diego Diaz & clerigo & Selidon de Ençiso & quantyoso \\
\hline La de lope Diaz & duena & Pero Xil & no quantyoso \\
\hline Rodrigo Diaz & ydo & Celidon herrero & quantyoso \\
\hline Ruy Diaz & clerigo & Françisco barbero & familiar \\
\hline
\end{tabular}




\begin{tabular}{|c|c|c|c|}
\hline Floristan hortelano & quantyoso & Maestre Sancho & clerigo \\
\hline Pedro Castillo & quantyoso & Anton Lopez & dize ser hidalgo \\
\hline Lucas tejedor & quantyoso & Gonçalo de Cornago & ydo \\
\hline Pedro del Rio & quantyoso & La de Pablo & quantioso \\
\hline Pero Sanches & pobre & Myguel Palaçio & quantioso \\
\hline Myguel de Caragosa & quantyoso & Martyn de Arnedillo & quantioso \\
\hline Juan de Cornago & cabailero & Juan Breton & quantioso \\
\hline Pedro de Arze & dize ser hidalgo & Pedro de la Parra & quantioso \\
\hline La de Juan de Gil & quantyosa & Pedro de Olibar & dize ser hidalgo \\
\hline Gil Martynez & quantyoso & Martyn Serrano & quantioso \\
\hline $\begin{array}{l}\text { Pero Martynez } \\
\text { hierno de Diego }\end{array}$ & quantioso & $\begin{array}{l}\text { Anton Capata } \\
\text { Juan Hortiz }\end{array}$ & $\begin{array}{l}\text { dize ser hidalgo } \\
\text { do }\end{array}$ \\
\hline Pero Martynez & & Pero soguero & quantioso \\
\hline molynero & quantioso & Juan Rodrigues & dize ser hidalgo \\
\hline Juan de Erra & quantioso & Juan Ximenez & ydo \\
\hline Diego Morzin & quantyoso & Pero Ximenez & ydo \\
\hline Juan Martynez & hidalgo & Gil de la Parra & quantyoso \\
\hline Diego Martynez & hidalgo & Su hierno & quantyoso \\
\hline Alonso Carrillo & quantioso & Albaro Gimenez & ydo \\
\hline Juan de Arnedillo & quantioso & Diego Ximenez & ydo \\
\hline Rodrigo de Soba & ydo & Juan de Resa & quantioso \\
\hline Diego Breton & ydo & Lope de Morillo & quantyoso \\
\hline La de Garçia & & Martyn de Arnedo & dize ser hidalgo \\
\hline Martynez & duena & Aleco & pobre \\
\hline Juan Martinez & ydo & La de Martyn de & \\
\hline Espinosa & $\begin{array}{l}\text { dize que es } \\
\text { hidalgo }\end{array}$ & $\begin{array}{l}\text { Arnedillo } \\
\text { Juan Rodrigues }\end{array}$ & $\begin{array}{l}\text { duena } \\
\text { dize ser hidalgo }\end{array}$ \\
\hline Juan de Mena & dize ser hidalgo & La de Anton & \\
\hline La de Pero Lloreynte & duena & Lloreynte & dueno \\
\hline Juan Fernandez & ydo & Sebastian Castillo & dize ser hidalgo \\
\hline Juan Sanz & ydo & Su hierno Labres & dize ser hidalgo \\
\hline Diego Martyn & quantioso & Gonçalo de Reso & quantyoso \\
\hline Juan Sanz de & & Juan Redondo & quantyoso \\
\hline Entranbasaguas & dize ser hidalgo & Pedro de Ayala & dize ser hidalgo \\
\hline Juan de Resa & quantyoso & Ruy Garçia & dize ser hidalgo \\
\hline Su hierno & quantyoso & Myguel Peres & caballero \\
\hline Juan Diez & ydo & Anton de Hita & dize ser hidalgo \\
\hline Juan Morillo & dize ser hidalgo & La de Ximenez & duena \\
\hline Juan Sanches & dize ser hidalgo & Albaro Lloreynte & quantyoso \\
\hline Diego de Lirozque & dize ser hidalgo & La de Garçia Capata & duena \\
\hline Rodrigo de Bobadilla & ydo & Pero Zapata & hidalgo \\
\hline Juan de Resa & quantioso & Sancho Sanches & dize ser hidalgo \\
\hline Juan Martinez & ydo & Su hijo Martyn & \\
\hline Pero Lopez & quantioso & Sanchez & dize ser hidalgo \\
\hline Gonçalo Calamoço & quantioso & Garçia Sanz & ydo \\
\hline
\end{tabular}


Pedro de Bicastillo

Myguel Lopez

Diego Capata

la de Gonçalo de

Riquel

Lope ferrero

La de Ruy Diaz

Gil de Carracayo

Gil de Carracayo

Martyn Garçia

Garçia Lopez

juan Bermejo

La de Martyn Sanz

Pero Muro

Juan de Muro

Martyn de Muro

La de Juan Ramirez

Pedro perayle

Pasquala

Gil Palaçio

Martyn de Matute

Pero terrero

dalgo

Pedro de Nicolas

Alonso Albarez

Pasqual

Diego Remon

Santillan

Juan Sanz de Matute

El hilio de Diego

Marlynez

juan de Torres

Juan Moreno

Ruy Lopez

Juan Garcia

Juan de Corres

Juan Miguel

Su madre

Diego Adan

Horeynte Nabarrete

Martyn de Labentosa

Martyn del Alamaco

Pero Perez dize ser hidalgo

dize ser hidalgo

dize ser hidalgo

duena

pobre

duena

quantyoso

quantioso

quantioso

ydo

quantioso

duena

quantioso

quantyoso

quantyoso

duena

quantyoso

quantyoso

quantyoso

quantioso

dize que es hi-

dize ser hidalgo

dize ser hidalgo

quantioso

dize ser hidalgo

quantioso

dize ser hidalgo

ydo

dize ser hidalgo

quantioso

ydo

quantioso

clerigo

quantioso

quantyoso

quantioso

quantioso

quantioso

quantyoso

quantyoso

\section{Collación de SANTIAGO}

Diego Sanz de la

Mata

quantyoso

Pero Ximenez

texedor

Hernando Ruyz de

Tudelilla

Juan Ochoa

Juan de Bitorya

Juan Breton

Juan Hernesto

Sancho

La de Diego Myguel

Hernando de Cornago

Diego de Luys

Juan Entrena

Pero Ruyz de Cerbera

Juan Martynez de

Gedonys

Sancho Marinero

Pedro Brabo

Pedro Ramyrez

Gonzalo de Matute

La de Juan Moreno

Juan çapatero

Domyngo Perez

Pedro de Moreda

Hernando de

Bobadilla

Hernando Diaz

Hernando de Bannos

Diego de Billarroyo

Myguel de Braso

juan de Castro quantioso

dize que es

hidalgo

dize que es

hidalgo

dize que es

hidalgo

dize que es

hidalgo

pobre

non quantyoso

quantyosa

quantyoso

diz que es

hidalgo

diz que es

hidalgo

diz que es

hidalgo

hidalgo

diz ser hidalgo

quantyoso

hidalgo

hidalgo

quantyosa

pobre

quantyoso

hidalgo

diz que es

hidalgo

clerigo

clerigo

quantioso

pobre

diz que es

hidalgo 


\begin{tabular}{|c|c|c|c|}
\hline Juan de Neas & diz que es & Sancho Sanz de & \\
\hline & hidalgo & Paganduro & dis que es \\
\hline Diego Breton & $\begin{array}{l}\text { diz que es } \\
\text { hidalgo }\end{array}$ & Pedro de Marroquyn & $\begin{array}{l}\text { niadigo } \\
\text { dis que es }\end{array}$ \\
\hline Juan Breton & diz que es & & hidalgo \\
\hline & hidalgo & Juan Roldan & quantyoso \\
\hline Juan Gaston & & Françisco Gascon de & \\
\hline molynero & quantyoso & Tejada & diz que es \\
\hline Pero Gimenez & quantyoso & & hidalgo \\
\hline Sancho de Argueda & quantyoso & Françisco Gascon de & \\
\hline Martyn Cornago & quantyoso & Hazedo & quantyoso \\
\hline Pedro el carretero & quantyoso & Salazar & quantyoso \\
\hline Diego Albarez & $\begin{array}{l}\text { dize que es } \\
\text { hidalgo }\end{array}$ & $\begin{array}{l}\text { Toda Lopez } \\
\text { Juan de Muro }\end{array}$ & $\begin{array}{l}\text { duena } \\
\text { quantyoso }\end{array}$ \\
\hline Pero Ruyz del Rincon & quantyoso & Juan de Muro el moço & quantyoso \\
\hline Juan de Hita & $\begin{array}{l}\text { dize que es } \\
\text { hidalgo }\end{array}$ & $\begin{array}{l}\text { La de Juan de Muro } \\
\text { Markyn Ardon }\end{array}$ & $\begin{array}{l}\text { quantyosa } \\
\text { quantyoso }\end{array}$ \\
\hline Hita escudero & honbre de armas & Myguel Serrano & quantyoso \\
\hline Myguel Brabo & familiar & Martyn de & \\
\hline Juan Diaz su hierno & quantyoso & Monteagudo & quantyoso \\
\hline Su hijo de Juan del & & Martin Garçon & quantyoso \\
\hline Muro & quantyoso & Juan Gatendo & quantyoso \\
\hline Pero Fernandez & quantyoso & Juan de Paredes & diz que es \\
\hline Pedro Calbo & quantyoso & & hidalgo \\
\hline Juan de Medrano & & Myguel Marin & quantyoso \\
\hline tanborin & $\begin{array}{l}\text { diz que es } \\
\text { hidalgo }\end{array}$ & $\begin{array}{l}\text { Juan Marin } \\
\text { Juan Garçia }\end{array}$ & clerigo \\
\hline Diego Santoro & clerigo & cintero & dis que es \\
\hline Su cunada & quantiosa & & hidalgo \\
\hline Sus hijos & quantyosos & Pedro de Basarita & quantyoso \\
\hline Pedro de Solorçeno & pobre & La de Pero Calbo & quantyoso \\
\hline La de Sancho Sanches & duena & Pero Gomez & clerigo \\
\hline Juan de Cornago & $\begin{array}{l}\text { diz que es } \\
\text { hidalgo }\end{array}$ & $\begin{array}{l}\text { La de Juan de Castro } \\
\text { Gil Maçotero }\end{array}$ & $\begin{array}{l}\text { quantyosa } \\
\text { quantyoso }\end{array}$ \\
\hline Diego Martinez & quantyoso & Albaro de San Juan & quantyoso \\
\hline Pedro de Carracayo & quantyoso & Martin de Camora & quantyoso \\
\hline Juan Baston el mayor & quantyoso & Maria Rodrigues & pobre \\
\hline La de Myguel Garçia & duena & Gonçalo de Grabalos & quantyoso \\
\hline $\begin{array}{l}\text { Pedro de Bronda } \\
\text { La de Pedro de }\end{array}$ & quantyoso & Rodrigo de Jubera & $\begin{array}{l}\text { diz que es } \\
\text { hidalgo }\end{array}$ \\
\hline Arnedo & dis que es & Diego Perez çapatero & quantyoso \\
\hline & duena & Pero Gomez & quantyoso \\
\hline Luys de Paredes & diz que es & Pero Ruyz sastre & quantyoso \\
\hline & hidalgo & La de Pedro Esquerro & quantyoso \\
\hline Pedro de Bas & & Myguel Marçilla & quantyoso \\
\hline pregonero & quantyoso & Pero Baston & quontyoso \\
\hline
\end{tabular}




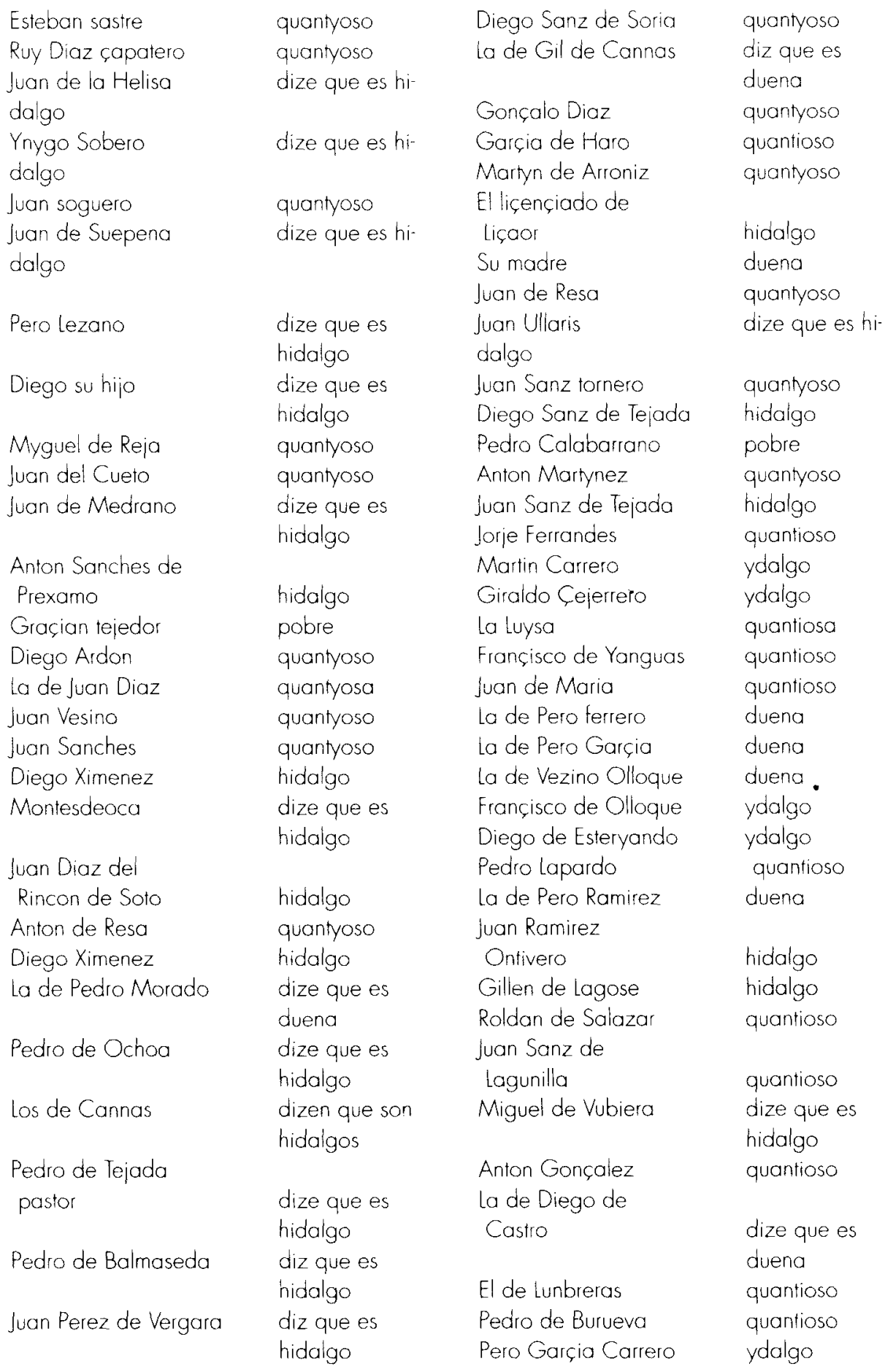




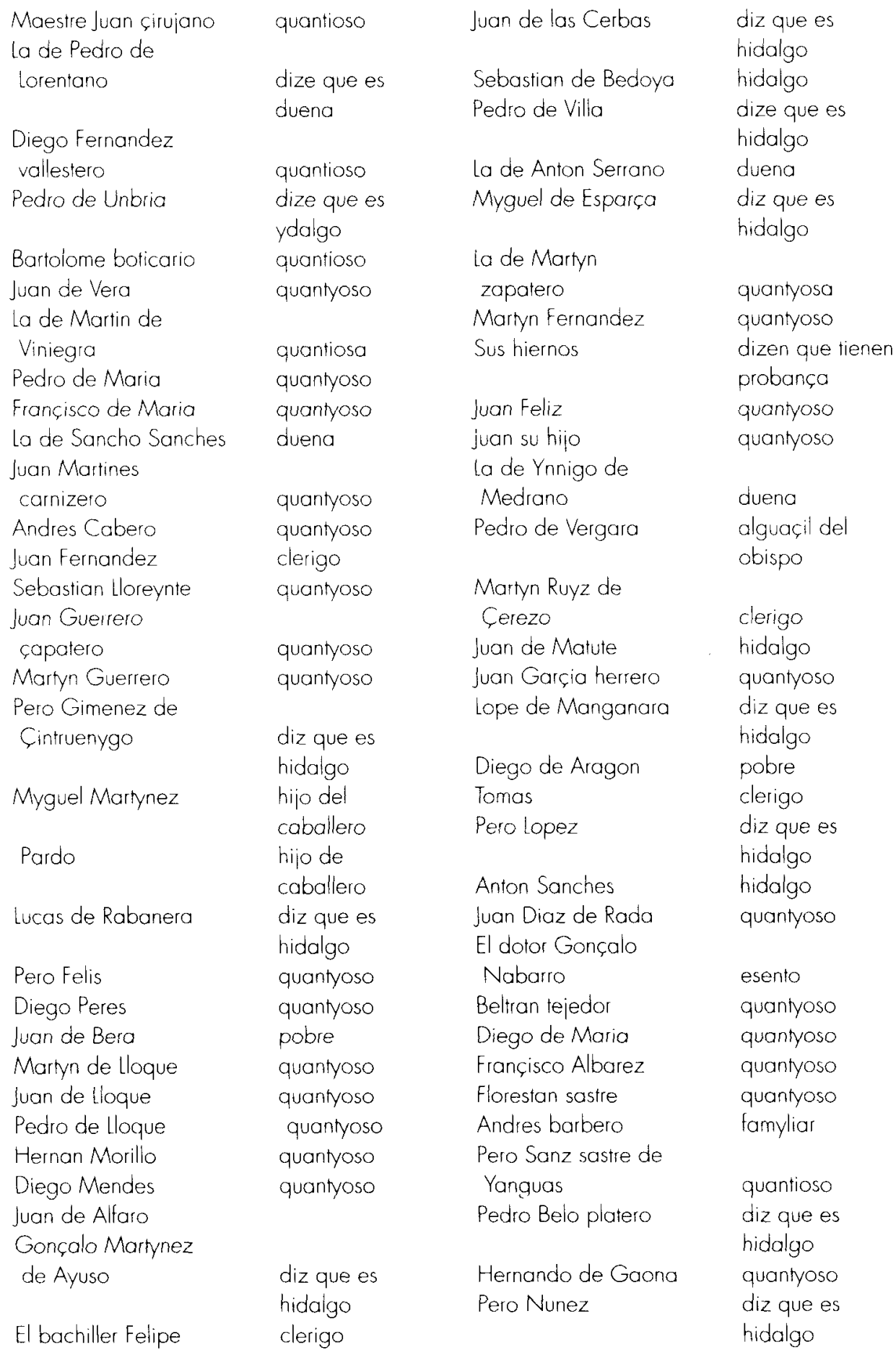




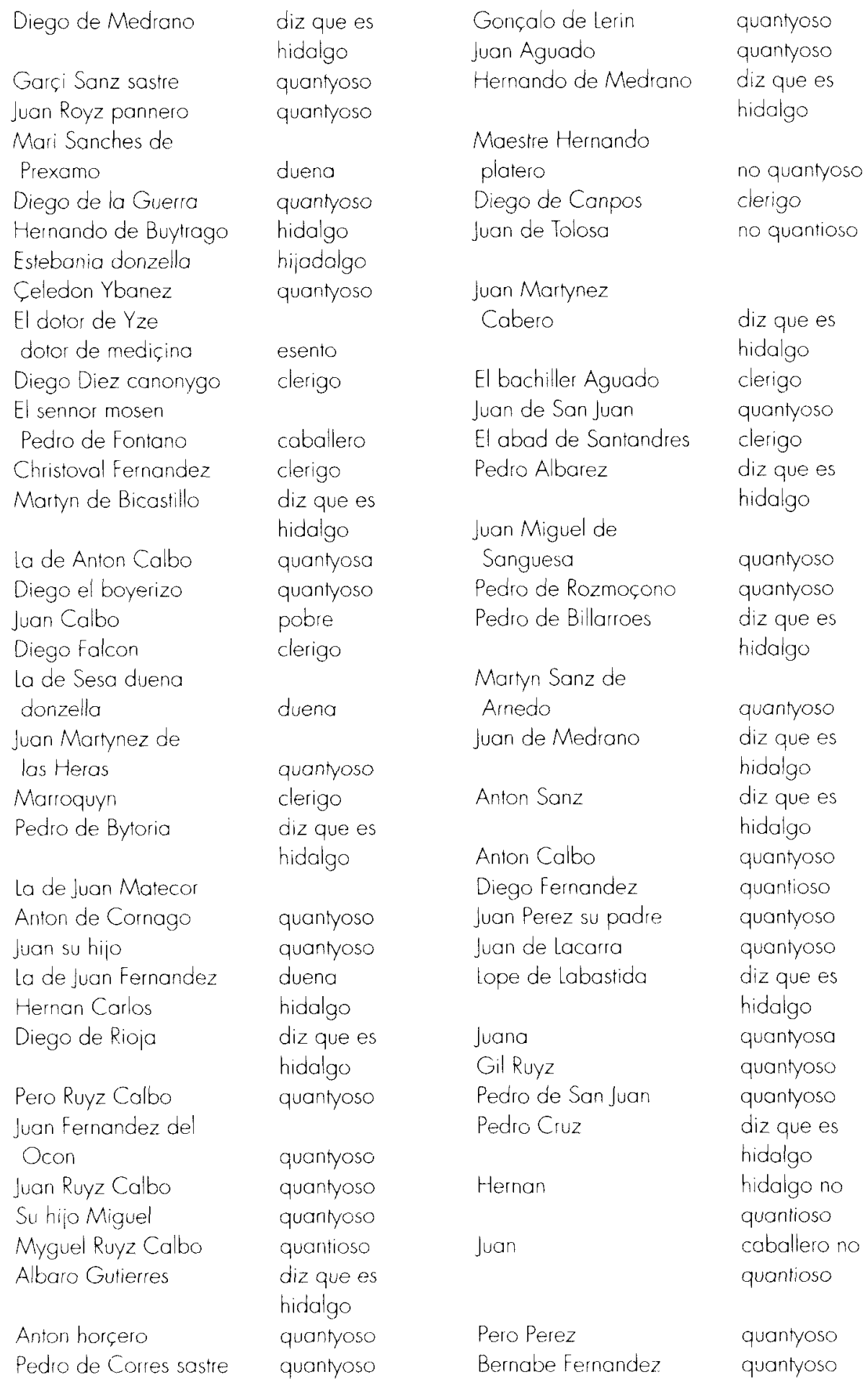




\begin{tabular}{|c|c|c|c|}
\hline La de Martyn Cordon & quantyoso & Celedon Roldan & clerigo \\
\hline Sancho Brabo & quantyoso & Pero Sanz Roldon & clerigo \\
\hline La de Pedro Vergara & duena & Su madre & \\
\hline Romero Barinque & hidalgo & El arçediano viejo & clerigo \\
\hline Alonso Lopez de & & Muto & clerigo \\
\hline Moriez & $\begin{array}{l}\text { diz que es } \\
\text { hidalgo }\end{array}$ & $\begin{array}{l}\text { Ferran Mayor } \\
\text { Teresa de Gamarra }\end{array}$ & $\begin{array}{l}\text { ydalgo } \\
\text { quantiosa }\end{array}$ \\
\hline Anton Gualite & quantyoso & Juan de Arronez & quantioso \\
\hline Gonçalo Munoz & hidalgo & Su madre & \\
\hline Hijo de Coled moro & quantyoso & Miguel hornero & no quantioso \\
\hline Su suegra & $\begin{array}{l}\text { dize que es } \\
\text { duena }\end{array}$ & $\begin{array}{l}\text { Françisco Prieto } \\
\text { Pedro de Santa Cruz }\end{array}$ & $\begin{array}{l}\text { no quantioso } \\
\text { dize que es }\end{array}$ \\
\hline Teresa su hermana & $\begin{array}{l}\text { diz que es } \\
\text { hidalgo }\end{array}$ & La de Pedro Beltran & $\begin{array}{l}\text { hidaligo } \\
\text { quantiosa }\end{array}$ \\
\hline Juan del Cano & quantyoso & El rerrador & no quantioso \\
\hline $\begin{array}{l}\text { Gonçalo Sanchez de } \\
\text { Laguiman }\end{array}$ & hidalgo & $\begin{array}{l}\text { El maestro de la } \\
\text { capilla }\end{array}$ & clerigo \\
\hline $\begin{array}{l}\text { Gil Morillo } \\
\text { La de Juan } \\
\text { Rodrigues de }\end{array}$ & quantyoso & $\begin{array}{l}\text { El yerno de la } \\
\text { palençiana ferrador } \\
\text { Juan de Vergara }\end{array}$ & quantioso \\
\hline Byniegra & quantyoso & sastre & quantioso \\
\hline $\begin{array}{l}\text { La de Sancho del } \\
\text { Cano }\end{array}$ & quantyoso & $\begin{array}{l}\text { Françisco de Vergara } \\
\text { Pero Perez de }\end{array}$ & quantyoso \\
\hline $\begin{array}{l}\text { Bartol del Cano } \\
\text { La de Pero Calbo }\end{array}$ & $\begin{array}{l}\text { quantyoso } \\
\text { diz que es } \\
\text { dueno }\end{array}$ & $\begin{array}{l}\text { Yanguas } \\
\text { La de Pero Martynez } \\
\text { Graçion sastre }\end{array}$ & $\begin{array}{l}\text { clerigo } \\
\text { no quantyosa } \\
\text { quantyoso }\end{array}$ \\
\hline Juan Sanz de Tejada & & Diego de Agusejo & quantyoso \\
\hline escribano & idalgo & Diego de Lerin & dize que es \\
\hline Pedro de Ceballos & clerigo & & hidalgo \\
\hline Juan de Segura & diz que es & Pedro de Ençiso & quantyoso \\
\hline & hidalgo & Ximenez & no quantyoso \\
\hline Antonio de Obiedo & diz que es & Hernandez pregonero & quantyoso \\
\hline & hidalgo & Sebastian Sanches & quantyoso \\
\hline Romero Guded & quantyoso & Françisco de Artiaga & quantyoso \\
\hline $\begin{array}{l}\text { Andres molynero } \\
\text { Juan Martynez de }\end{array}$ & quantyoso & $\begin{array}{l}\text { Pedro Montesino } \\
\text { dalgo }\end{array}$ & dize que es hi- \\
\hline Prexamo & quantyoso & La de Juan Diaz & \\
\hline Pero Coxa & quantyoso & perayle & quantiosa \\
\hline Su hierno & quantyoso & El liçençiado de & \\
\hline Pero Leza & quantioso & Lacamen & clerigo \\
\hline La de Juan el moredo & quantioso & Juan de Hieracheta & quantyoso \\
\hline Juan de Medrano & $\begin{array}{l}\text { dize que es } \\
\text { hidalgo }\end{array}$ & $\begin{array}{l}\text { Diego Perez capatero } \\
\text { Juan Martinez de }\end{array}$ & quantyoso \\
\hline Graçia la tendera & quantiosa & Ençiso & clerigo \\
\hline Su nuera & quantiosa & & \\
\hline
\end{tabular}


El liçençiado de la gramatica

\section{ALDEANUEVA}

\section{Gonçalo Rascon}

Pero Martinez de

Cornago

Pero Diaz

Martin

Sarrascon

La de Pero Morilio

Pero Perez hijo de

Martyn Peres

Pero Perez hijo de

Juan Perez

La de Bartol Perez

La de Martyn Ruyz

Martyn Perez

Gil Perez su hijo

Pero Ximenez

Juan Perez su

hermano

Gil Martynez hijo

de Martyn Ruyz

Pero Rascon

Martin Roldan

Pedro de Arze

Lope Marcilla

Juan Ruyz su hierno

Gil Perez

Pero Martynez

Hernan Marrodan

Pero Roldan

Pero Ortega

Diego Marin

Herederos de Martyn

Diaz

Bartol Gimenez el

moço

juan pastor

Juan Ybanez clerigo

quantyoso

clerigo

quantyoso

quantyoso

quantyoso

quantyoso

quantioso

quantioso

quantyoso

quantyoso

quantyoso

quantyoso

quantyoso

quantyoso

quantyoso

quantyoso

quantyoso

quantyoso

quantyoso

quantyoso

quantyoso

quantyoso

quantyoso

quantyoso

quantyoso

quantyoso

quantyosos

quantyoso

quantyoso

quantyoso
Pero Ximenez de

Grabalos

Pero Ybanez

Juan Martinez

Anton Gutierrez

Martyn Ruyz

Martin Ruyz

Diego Antonio

Juan Marçilla

Diego Gutierrez

Myguel Ybanez

Diego Bonero

Pero Gimenez

Pero Finada

Gonçalo Ruyz

La bihuda de Juan

Sanz

Diego Perez

Los herederos de

Antonio Hortega

Juan de Herrada

Anton Moreno

Pero de Arnedo

La de Juan Marçilla

biuda

juan Perez su hijo

Martyn Perez su hijo

Martyn Marçilla

Juan Fernandez

Anton Ruyz

Juan Ruyz

Alonso Ruyz su hijo

Anton de la Cuesto

Juan Ruyz Nabarro

Gil Perez el sordo

Gil herrero

Martyn de la Cuesta

Juan de Nagori

Myguel Blazquez

Sus sobrinos

Hernan Perez

La de Gil Perez

Juan pastor quantyoso

clerigo

quantyoso

quantyoso

quantyoso

quantyoso

dize que es

hidalgo

quantyoso

quantyoso

quantyoso

quantyoso

quantyoso

quantyoso

quantyoso

quantyosa

quantyoso

quantyosos

dize que es

hidalgo

quantyoso

quantyoso

quantyoso

quarityoso

quantyoso

quantyoso

quantyoso

quaniyoso

quantyoso

quantyoso

quantyoso

quantyoso

quantyoso

quantyoso

quantyoso

clerigo

quantyoso

quantyosos

quantyoso

quantyoso

quantyoso 


\begin{tabular}{|c|c|c|c|}
\hline Martyn de Vergara & quantyoso & Pedro Hortega & quantyoso \\
\hline Juan Gutierrez & quantyoso & Gonçalo Ximenez & quantyoso \\
\hline Gil Perez & quantyoso & Juan Ruyz & clerigo \\
\hline Pedro de Lara & quantyoso & Anton Maya & quantyoso \\
\hline Juan Calleja & quantyoso & Pero Marçilla & quantyoso \\
\hline Juan Moreno & quantyoso & Sebastian Sanches & quantyoso \\
\hline Pero Ruyz & quantyoso & Diego Sanches & quantyoso \\
\hline Juan Ruyz & quantyoso & Garçia Perez & quantyoso \\
\hline Martyn Perez & quantyoso & Martyn Perez & quantyoso \\
\hline Viçente & quantyoso & La criada de Martyn & \\
\hline Bartol Perez & quantyoso & Perez & quantyosa \\
\hline Alonso Gutierres & & Pero Gutierrez & quantyoso \\
\hline Moreno & quantyoso & Pero Merino & quantyoso \\
\hline Pedro Adan & quantyoso & Martin Gutierres & quantyoso \\
\hline Hernan Martynez & quantyoso & Hernan Perez & quantioso \\
\hline Juan Martinez & quantyoso & Martyn Sanches & quantyoso \\
\hline Martyn Manzebo & quantyoso & Juan Ruyz & quantyoso \\
\hline Juan Garçia & quantyoso & Juan Ximenez & quantyoso \\
\hline Juan Gimenez & quantyoso & juan de la Aldea & quantyoso \\
\hline Juan Ximenez & quantyoso & Antonio Ybanez & quantyoso \\
\hline Sancho de Aranda & dize que es & Juan de Villarroya & quantyoso \\
\hline & hidalgo & Myguel Ximenez & quantyoso \\
\hline Juan de Autol & quantyoso & Anton del Muro & quantyoso \\
\hline Martyn Hortega & quantyoso & Barolome Roldan & quantyoso \\
\hline Myguel de San & & Pedro pastor & quantyoso \\
\hline Myguel & quantyoso & Herederos de Myguel & \\
\hline Gil Sanches & quantyoso & Perez & quantyosos \\
\hline Martyn Adan & quantyoso & Juan criado de Andres & quantioso \\
\hline Pero Gimon & quantyoso & Anton pastor moço & \\
\hline Diego Miiranda & quantyoso & de Pero Perez & quantioso \\
\hline Myguel de la Mata & quantyoso & Diego de tera moco & quantyoso \\
\hline Juan de Muro & quantyoso & Los dichos Martin & \\
\hline Hernan Martinez & quantyoso & Perez, Juan Calleja & \\
\hline Pero Ximenez & quantyoso & Martyn Garçia & \\
\hline Diego Marcilla & quantyoso & Carrera, Juan Ruyz & \\
\hline Juan Perez de Lanyeta & quantioso & panero & \\
\hline Myguel de la Aldea & quantioso & & \\
\hline $\begin{array}{l}\text { Juan Benyte } \\
\text { Juan Ybanez y }\end{array}$ & quantyoso & & \\
\hline Bicente & quantyoso & MURILLO & \\
\hline Pero Lardo & quantyoso & & \\
\hline Myguel Lardo & quantioso & & \\
\hline Myguel del Oro & quantyoso & Juan Royo & quantyoso \\
\hline La de Villarroya & quantyoso & Juana Garçia & quantyosa \\
\hline Pedio de la Cuesta & quantyoso & Juan Hernanz & quantyoso \\
\hline
\end{tabular}




\begin{tabular}{|c|c|c|c|}
\hline Juan Myguel & quantyoso & Juan Luso & quantyoso \\
\hline Juan de Bea & quantyoso & Alonso Diaz de & \\
\hline Juan de Arnedo & quantyoso & Juan Martyn & quantyoso \\
\hline Sancho Martinez & quantyoso & Juan Martyn de & \\
\hline Pedro de Tejada & dize ser hidalgo & Carbonera & clerigo \\
\hline Juan Alcalde & quantyoso & Pedro Roman & quantyoso \\
\hline Christobal Vesino & quantyoso & Juan tabernero & quantyoso \\
\hline Juan Martynez & & Alonso de Piya & disze que es $h$ \\
\hline el biejo & quantyoso & & idalgo \\
\hline Pero Ximenez & quantyoso & & \\
\hline Diego Moreno & quantyoso & \multirow{3}{*}{\multicolumn{2}{|c|}{$\begin{array}{l}\text { Estaban al pie de este dicho padron dos } \\
\text { fermas e en ellas dos nonbres que desçian } \\
\text { Juan Marçillo, Juan Royz panero }\end{array}$}} \\
\hline Juan Moreno & quantyoso & & \\
\hline Diego Adan & quantyoso & & \\
\hline Andres Sanches & quantyoso & Diego Gil & quantyoso \\
\hline Los herederos de & & Juan de la Nueba & quantyoso \\
\hline Diego Cordon & quantyosos & Juan Sanz & quantyoso \\
\hline Juan Alcalde & quantyoso & Juan Merino & quantyoso \\
\hline Garçia Martynez & quantyoso & La de Pero Garcia & quantyoso \\
\hline Pero Aguado & quantyoso & Sancho Vesino & quantyoso \\
\hline Diego Perez & quantyoso & La de Secadura & duena \\
\hline Juan Domynguez & quantyoso & Juan Ybanez & quantyoso \\
\hline La de Hernan & & Diego Ruyz & quantyoso \\
\hline Martinez & quantyoso & La de Rodrigo & \\
\hline Juan Martinez & quantyoso & barbero & quantyoso \\
\hline Gonçalo Martynez & quantyoso & Diego Narrodan & quantyoso \\
\hline Martyn Serrano & quantyoso & La de Pijo & quantyoso \\
\hline Andres de Tera & quantyoso & La de Hernan & \\
\hline Martyn de Arpano & quantyoso & pastor & quantyoso \\
\hline Myguel de Pedro & quantyoso & Juan Ximenez & quantyoso \\
\hline Diego Resçio & quantyoso & Pero Sanz Feliz & quantyoso \\
\hline Juan de Ençiso & quantyoso & Juan Miranda & quantyoso \\
\hline Hernan Martynez & quantyoso & Alonso de Bera & quantyoso \\
\hline Gonçalo Monyila & quantyoso & La de Martyn Ruyz & quantyoso \\
\hline Sancho Myguel & quantyoso & Pero Ruyz su hijo & quantyoso \\
\hline Pero Martynez & quantioso & Miguel Solano & quantyoso \\
\hline Diego de Corre & quantyoso & Juan Solano & quantyoso \\
\hline Juan pellexero & quantyoso & Alonso de Corre & quantyoso \\
\hline Pedro Adan & quantyoso & Pero Diaz & quantyoso \\
\hline Pedro Corre & quantyoso & Pero Monylla & quantyoso \\
\hline Andres de Jubera & dize que es & La de Juan herrero & quantyoso \\
\hline & hidalgo & Diego Magaso & pobre \\
\hline Pero Adan el moço & quantioso & Miguel del Criado & quantyoso \\
\hline Anton Solomon & quantyoso & Pero escudero & dize que es \\
\hline Diego el Rogo & quantyoso & & hidalgo \\
\hline Bartollades & clerigo & Pero Moreno & quantyoso \\
\hline Lo de Roarta & quantyosa & Rodrigo Ruyz & quantyoso \\
\hline
\end{tabular}




\section{juan de Torres \\ Pero Franco el moço \\ Martyn Salazar \\ Juan barbero \\ Myguel de Arasa}

\section{RINCÓN DE SOTO}

Diego Marin
Juan Martynez
Diego de Miguel
Juan Carrillo
Juan de Arnedillo
Pedro de Bobadilla
Diego Benyto
La de Gonçalo
Martynez
Juan Martynez

Espinosa

Juan de Mena

La del platero

juan fornero

juan Sanz

juan Sanz

Juan de Sarresa

Juan Merino

Juan Diaz

Juan Morillo

Juan Gomez

Diego de

Hernanmaityn

juan de Resa

Juan Martinez

Pero Lopez

Gonçalo de Almagen

Maestre Sancho

quantyoso
quantyoso
quantyoso
quantyoso
dize ser hidalgo

quantyoso

hidalgo

hidalgo

quantyoso

quantyoso

ydo

ydo

duena

ydo

dize que es

hidalgo

dize que es

hidalgo

duena

dize que es

hidalgo

ydo

dize que es

hidalgo

quantyoso

quantyoso

ydo

quantyoso

diz que es

hidalgo

dize que es

hidalgo

quantyoso

ydo

quantyoso

quantyoso

quantyoso
Anton Lopez

Gonçalo de Cornago

La de Pablo

Myguel Palaçio

Juan Lloreynte

Pedro de la Parra

Martyn Serrano

Anton Zarraga

Juan Hortiz

Pero soguero

Juan Sanches

Juan Martyn

Pero Ximenez

Gil de la Parra

Su hierno

Albaro Gimenez

Juan de Resa

Lope Morillo

Juan de Arnedo

Hillero

La de Martyn Sanz

Marcilla

Juan Rodrigues

La de Anton Benyto

Sebastian de

Astudilio

Garcia Moreno

Gonçalo de Reso

Juan Redondo

Pedro de Hiela

Ruy Garcia

Myguel Rey

Myguel de Hita

Anton de Hita

La de Ramon

Fernandez

Albaro Lloreynte

La de Garcia

Pero Caparra

Sancho Gonçalez quantyoso

ydo

quantyoso

quantyoso

quantyoso

quantyoso

quantyoso

dize ser hidalgo

ydo

quantyoso

quantyoso

ydo

ydo

quantyoso

quantyoso

ydo

quantyoso

dize que es

hidalgo

dize que es

hidalgo

pobre

duena

diçe que es

hidalgo

duena

dize ser hidalgo

dize ser hidalgo

quantyoso

quantyoso

dize ser hidalgo

dize ser hidalgo

caballero

dize ser hidalgo

dize ser hidalgo

duena

quantyoso

duena

ydo

dize ser hidalgo 
Su hijo Martyn

Gonçalez

Garcia Sanz

Pedro de Autylio

Myguel Lopez

Diego Gasaya

La de Gonçalo de

Lasoy
Lope herrero

Martyn de Medina

La de Ruy Diaz

Gil de Tamayo

Martyn Garçia

Gonçalo Lopez

Juan Vermejo

La de Martyn Sanz

Pero Martin

Juan de Mero

Martyn Serrano

La de Juan Feliz

Pero Pelayz

Pasqual Alonso

Gil Pelayo dize ser hidalgo

dize ser hidalgo

dize ser hidalgo

dize ser hidalgo

dize ser hidalgo

duena

pobre

pobre

duena

quantyoso

quantyoso

ydo

quantyoso

duena

quantyoso

quantyoso

quantyoso

duena

quantyoso

quantyoso

quantyoso
Martyn de Matute

Pero Feliz

Alcarre

Pero Martin

Diego Feno

Sahurielo

Juan Sanz de Matute

Juan de Corres

Juan Moreno

Ruy Lopez

Juan Garçia

Juan Torres

Juan Gutierrez

Juan Myguel

Su madre

Diego Adan

Pero Perez

Martyn de Nabarrete

Ynygo

Martyn de Labentosa

Martyn el moço quantyoso

dize ser hidalgo

disze ser

hidalgo

quantyoso

disze ser

hidalgo

quantyoso

dize ser hidalgo

dize ser hidalgo

quantyoso

ydo

quantyoso

clerigo

clerigo

quantyoso

quantyosa

quantyoso

quantyoso

quantyoso

quantyoso

quantyoso

quantyoso 